ESAIM: M2AN 46 (2012) 1147-1173

DOI: $10.1051 / \mathrm{m} 2 \mathrm{an} / 2011075$
ESAIM: Mathematical Modelling and Numerical Analysis

www.esaim-m2an.org

\title{
CONVERGENCE OF SOME ADAPTIVE FEM-BEM COUPLING FOR ELLIPTIC BUT POSSIBLY NONLINEAR INTERFACE PROBLEMS
}

\author{
Markus Aurada $^{1}$, Michael Feischl ${ }^{1}$ and Dirk Praetorius ${ }^{1}$
}

\begin{abstract}
We consider the symmetric FEM-BEM coupling for the numerical solution of a (nonlinear) interface problem for the 2D Laplacian. We introduce some new a posteriori error estimators based on the $(h-h / 2)$-error estimation strategy. In particular, these include the approximation error for the boundary data, which allows to work with discrete boundary integral operators only. Using the concept of estimator reduction, we prove that the proposed adaptive algorithm is convergent in the sense that it drives the underlying error estimator to zero. Numerical experiments underline the reliability and efficiency of the considered adaptive mesh-refinement.
\end{abstract}

Mathematics Subject Classification. 65N30, 65N15, 65N38.

Received February 25, 2011. Revised August 22, 2011.

Published online February 13, 2012.

\section{INTRODUCTION AND OVERVIEW}

The $(h-h / 2)$-error estimation strategy is a well-known technique for the a posteriori estimation of the error in the energy norm $\left\|u-u_{\ell}\right\|$; see [23] in the context of ordinary differential equations, and the overview article of Bank [6] or the monograph [1], Chapter 5, in the context of the finite element method: let $\mathcal{X}_{\ell}$ be a discrete subspace of the energy space $\mathcal{H}$ and let $\widehat{\mathcal{X}}_{\ell}$ be its uniform refinement. With the corresponding Galerkin solutions $\mathbf{U}_{\ell}$ and $\widehat{\mathbf{U}}_{\ell}$, the $(h-h / 2)$-error estimator

$$
\eta_{\ell}:=\left\|\widehat{\mathbf{U}}_{\ell}-\mathbf{U}_{\ell}\right\|
$$

is a computable quantity [15] which can be used to estimate the error $\left\|\mathbf{u}-\mathbf{U}_{\ell}\right\|$, where $\mathbf{u} \in \mathcal{H}$ denotes the exact solution and where $\|\cdot\| \mid \|$ denotes the energy norm on $\mathcal{H}$.

For finite element methods (FEM), the energy norm, e.g., $\|\cdot\|\|=\| \nabla(\cdot) \|_{L^{2}(\Omega)}$ provides local information, which elements of the underlying mesh should be refined to decrease the error effectively. For boundary element methods (BEM), the energy norm $\|\cdot\|$ is (equivalent to) a fractional order Sobolev norm and typically does not provide a direct information, where the underlying mesh should be refined. In [20], localized variants of $\eta_{\ell}$ were introduced. In $[18,19]$ the equivalence of $\eta_{\ell}$ to hierarchical two-level error estimators from $[24,28]$ and averaging error estimators from [9-11] has been analyzed.

\footnotetext{
Keywords and phrases. FEM-BEM coupling, a posteriori error estimate, adaptive algorithm, convergence.

1 Institute for Analysis and Scientific Computing, Vienna University of Technology, Wiedner Hauptstraße 8-10, 1040 Wien, Austria. Markus.Aurada@tuwien.ac.at; Michael.Feischl@tuwien.ac.at; Dirk.Praetorius@tuwien.ac.at
} 
Recently [21], convergence of some $(h-h / 2)$-steered adaptive mesh-refinement has been proven for linear model problems in the context of FEM and BEM. In $[2,4]$, this result has been generalized to averaging type error estimators and perturbed Galerkin schemes. The latter is important in the context of BEM, since the involved integral operators can, in general, only be evaluated for discrete functions.

In this work, we introduce some $(h-h / 2)$-type error estimators for the coupling of FEM and BEM. As model problem, we consider an interface problem in two dimensions with a nonlinear inhomogeneous partial differential equation (PDE) in the interior domain and a linear homogeneous PDE in the exterior domain. We apply a symmetric coupling method $[13,14]$ and the lowest-order Galerkin scheme to obtain a (nonlinear) system of coupled FEM-BEM equations. For linear problems, the ideas from $[18,19]$ can be used to prove that the introduced error estimators are equivalent to the two-level error estimator from [27].

Using ideas from [2] and in addition to the seminal work [27], we include the approximation of the data to deal with discrete integral operators only. Moreover, [27] proves that a saturation condition

$$
\left\|\mathbf{u}-\mathbf{U}_{\ell+1}\right\| \leq q\left\|\mathbf{u}-\mathbf{U}_{\ell}\right\|
$$

for a sequence $\mathbf{U}_{\ell}$ of discrete FEM-BEM solutions and with some uniform constant $0<q<1$, implies the reliability of the two-level error estimator. On the other hand, this saturation condition already assumes linear convergence of the discrete solutions obtained from an adaptive mesh-refining algorithm. In our work, we assume that uniform refinement, i.e. the use of $\widehat{\mathbf{U}}_{\ell}$ instead of $\mathbf{U}_{\ell+1}$ in (1.2), guarantees a saturation condition. Under this - compared to [27] - much weaker assumption, we prove that the introduced $(h-h / 2)$-error estimator is reliable and efficient up to data approximation terms, which are also controlled a posteriori. Finally, we prove that the usual adaptive algorithm drives the error estimator (and hence the error) to zero. We stress that this is the first convergence result available for adaptive schemes in the context of the FEM-BEM coupling.

The outline of the paper is as follows: in Section 2.1, we formulate our model problem, and the Galerkin formulation is given in Section 2.2-2.3. Section 3.1 collects the properties of the local mesh-refinement used for the numerical analysis. In Section 3.2, we introduce a computable data oscillation term $\mathrm{osc}_{\ell}$. We prove that osc $\ell$ provides the means to control the error introduced by the data approximation (Prop. 3.1). In Section 3.3, we state and discuss the saturation assumption (3.13) and prove that the $(h-h / 2)$-error estimator $\eta_{\ell}$ from (1.1) provides, up to $\mathrm{osc}_{\ell}$, a lower and upper bound for the error $\left\|\mathbf{u}-\mathbf{U}_{\ell}\right\|$ (Prop.3.7). As mentioned before, the boundary contribution to the energy norm $\|\cdot\| \cdot \|$ cannot be used to steer an adaptive mesh-refinement. In the spirit of [20], we introduce further estimators $\mu_{\ell}$ and $\widetilde{\mu}_{\ell}$ in Section 3.4, which are equivalent to $\eta_{\ell}$ (Lem. 3.8) and which can be used to steer an adaptive algorithm. Consequently (Thm. 3.9), these estimators provide, up to osc $\ell$, lower and upper bounds for the error. Section 4 provides our version of the adaptive algorithm (Algorithm 4.4) and proves convergence (Thm. 4.5). The first ingredient of our convergence proof is the observation that adaptive mesh-refinement always leads to a convergent sequence of discrete solutions $\mathbf{U}_{\ell}$, where the limit

$$
\mathbf{u}_{\infty}:=\lim _{\ell \rightarrow \infty} \mathbf{U}_{\ell}
$$

however, does not necessarily coincide with the continuous solution $\mathbf{u}$ (Prop. 4.2). Second, we show that a generalized variant (4.4)-(4.5) of the Dörfler marking [16] implies an estimator reduction estimate of the type

$$
\widetilde{\varrho}_{\ell+1}^{2} \leq \kappa \widetilde{\varrho}_{\ell}^{2}+C\left\|\widehat{\mathbf{U}}_{\ell+1}-\widehat{\mathbf{U}}_{\ell}\right\|^{2}
$$

with $\widetilde{\varrho}_{\ell}^{2}:=\widetilde{\mu}_{\ell}^{2}+\operatorname{osc}_{\ell}^{2}$ and with certain $\ell$-independent constants $0<\kappa<1$ and $C>0$ (Lem. 4.3). From the a priori convergence (1.3) of $\widehat{\mathbf{U}}_{\ell}$ to some limit $\widehat{\mathbf{u}}_{\infty}$, one may thus conclude convergence $\widetilde{\varrho}_{\ell} \rightarrow 0$ as $\ell \rightarrow \infty$, $c f$. [4]. Section 5 gives empirical evidence that the proposed adaptive algorithm is much superior to uniform mesh-refinement with respect to both, experimental convergence rate and computational time. Finally, a short appendix generalizes a result of [13] and proves that, without any further assumptions on the mesh-sizes, each discrete space $\mathcal{X}_{\ell}$ admits a unique Galerkin solution $\mathbf{U}_{\ell}$. 


\section{Continuous problem and Galerkin Formulation}

\subsection{Model problem}

We consider the nonlinear interface problem

$$
\begin{cases}-\operatorname{div}\left(A \nabla u^{\mathrm{int}}\right)=f & \text { in } \Omega^{\mathrm{int}}:=\Omega, \\ -\Delta u^{\mathrm{ext}}=0 & \text { in } \Omega^{\mathrm{ext}}:=\mathbb{R}^{2} \backslash \bar{\Omega} \\ u^{\mathrm{int}}-u^{\mathrm{ext}}=u_{0} & \text { on } \Gamma, \\ \left(A \nabla u^{\mathrm{int}}-\nabla u^{\mathrm{ext}}\right) \cdot n=\phi_{0} & \text { on } \Gamma, \\ u^{\mathrm{ext}}(x)=\mathcal{O}(\log |x|) & \text { as }|x| \rightarrow \infty .\end{cases}
$$

Here, $\Omega$ is a bounded Lipschitz domain in $\mathbb{R}^{2}$ with polygonal boundary $\Gamma:=\partial \Omega$ and outer unit normal vector $n$. The given data satisfy $f \in L^{2}(\Omega), u_{0} \in H^{1 / 2}(\Gamma)$, and $\phi_{0} \in H^{-1 / 2}(\Gamma)$. We recall that $\widetilde{H}^{-1}(\Omega)$ is the dual space of $H^{1}(\Omega)$ with respect to the $L^{2}(\Omega)$-scalar product. The space $H^{1 / 2}(\Gamma)$ is precisely the space of all traces of functions from $H^{1}(\Omega)$, and $H^{-1 / 2}(\Gamma)$ is the dual of $H^{1 / 2}(\Gamma)$ with respect to the $L^{2}(\Gamma)$-scalar product.

As usual, (2.1) is understood in the weak sense, and the sought solutions satisfy $u^{\text {int }} \in H^{1}(\Omega)$ and $u^{\text {ext }} \in$ $H_{\ell o c}^{1}\left(\Omega^{\text {ext }}\right)=\left\{v: \Omega^{\text {ext }} \rightarrow \mathbb{R}: \forall K \subset \Omega^{\text {ext }}\right.$ compact $\left.v \in H^{1}(K)\right\}$ with $\nabla u^{\text {ext }} \in L^{2}\left(\Omega^{\text {ext }}\right)$. The (possibly nonlinear) operator $A: L^{2}(\Omega)^{2} \rightarrow L^{2}(\Omega)^{2}$ is strongly monotone and Lipschitz continuous, i.e. there holds

$$
\left\{\begin{array}{l}
C_{\text {mon }}\|\nabla v-\nabla w\|_{L^{2}(\Omega)}^{2} \leq\langle A \nabla v-A \nabla w, \nabla v-\nabla w\rangle_{\Omega}, \\
\|A \nabla v-A \nabla w\|_{L^{2}(\Omega)} \leq C_{\text {lip }}\|\nabla v-\nabla w\|_{L^{2}(\Omega)},
\end{array}\right.
$$

for all $v, w \in H^{1}(\Omega)$.

Problem (2.1) is equivalently stated via the symmetric FEM-BEM coupling, cf. e.g. [13], Theorem 1: find $(u, \phi) \in \mathcal{H}:=H^{1}(\Omega) \times H^{-1 / 2}(\Gamma)$ such that

$$
\left\{\begin{array}{l}
\langle A \nabla u, \nabla v\rangle_{\Omega}+\left\langle\mathfrak{W} u+\left(\mathfrak{K}^{\prime}-\frac{1}{2}\right) \phi, v\right\rangle_{\Gamma}=\langle f, v\rangle_{\Omega}+\left\langle\phi_{0}+\mathfrak{W} u_{0}, v\right\rangle_{\Gamma}, \\
\left\langle\psi, \mathfrak{V} \phi-\left(\mathfrak{K}-\frac{1}{2}\right) u\right\rangle_{\Gamma}=-\left\langle\psi,\left(\mathfrak{K}-\frac{1}{2}\right) u_{0}\right\rangle_{\Gamma},
\end{array}\right.
$$

for all $(v, \psi) \in \mathcal{H}$. Here, $\mathfrak{V}$ denotes the simple-layer potential, $\mathfrak{K}$ denotes the double-layer potential with adjoint $\mathfrak{K}^{\prime}$, and $\mathfrak{W}$ denotes the hypersingular integral operator. With

$$
G(z):=-\frac{1}{2 \pi} \log |z| \quad \text { for } z \in \mathbb{R}^{2} \backslash\{0\}
$$

the fundamental solution of the 2D Laplacian, these integral operators formally read for $x \in \Gamma$ as follows,

$$
\begin{aligned}
(\mathfrak{V} \psi)(x) & =\int_{\Gamma} G(x-y) \psi(y) \mathrm{d} \Gamma(y), \\
(\mathfrak{K} v)(x) & =\int_{\Gamma} \partial_{n(y)} G(x-y) v(y) \mathrm{d} \Gamma(y), \\
(\mathfrak{W} v)(x) & =-\partial_{n(x)} \int_{\Gamma} \partial_{n(y)} G(x-y) v(y) \mathrm{d} \Gamma(y) .
\end{aligned}
$$

By continuous extension, these definitions provide linear boundary integral operators $\mathfrak{V} \in$ $L\left(H^{-1 / 2}(\Gamma) ; H^{1 / 2}(\Gamma)\right), \quad \mathfrak{K} \in L\left(H^{1 / 2}(\Gamma) ; H^{1 / 2}(\Gamma)\right)$, and $\mathfrak{W} \in L\left(H^{1 / 2}(\Gamma) ; H^{-1 / 2}(\Gamma)\right)$ as well as $\mathfrak{K}^{\prime} \in L\left(H^{-1 / 2}(\Gamma) ; H^{-1 / 2}(\Gamma)\right)$. The reader is also referred to the monographs [25,30,31] for more details 
on these integral operators. By scaling of $\Omega$, we may assume that $\operatorname{diam}(\Omega)<1$ to ensure the uniform ellipticity of $\mathfrak{V}$, i.e.

$$
\|\psi\|_{H^{-1 / 2}(\Gamma)}^{2} \lesssim\langle\psi, \mathfrak{V} \psi\rangle_{\Gamma} \quad \text { for all } \psi \in H^{-1 / 2}(\Gamma) .
$$

The link between (2.1) and (2.3) is provided by $u=u^{\text {int }}$ and $\phi=\nabla u^{\text {ext }} \cdot n$, and $u^{\text {ext }}$ is then given by the third Green's formula

$$
u^{\text {ext }}(x)=\widetilde{\mathfrak{K}}\left(u-u_{0}\right)(x)-\widetilde{\mathfrak{V}} \phi(x) \text { for } x \in \Omega^{\text {ext }},
$$

where the potentials $\widetilde{\mathfrak{V}}$ and $\widetilde{\mathfrak{K}}$ formally denote the operators $\mathfrak{V}$ and $\mathfrak{K}$, but are now evaluated in $\Omega^{\text {ext }}$ instead of $\Gamma$. Note carefully that we do not use a notational difference for the function $u \in H^{1}(\Omega)$ and its trace $u \in H^{1 / 2}(\Gamma)$, for which we compute the boundary integrals $\mathfrak{W} u$ and $\left(\mathfrak{K}-\frac{1}{2}\right) u$ in $(2.3)$.

It is well-known that $(2.3)$ is well-posed in the sense that it allows for a unique solution $(u, \phi) \in \mathcal{H}$. Since we need some arguments from [13] below, we briefly recall the corresponding proof of [13], Corollary 2: first, the second equation of $(2.3)$ is equivalently written as

$$
\phi=\mathfrak{V}^{-1}\left(\mathfrak{K}-\frac{1}{2}\right)\left(u-u_{0}\right) .
$$

This identity may be used to eliminate $\phi$ in the first equation of (2.3). This gives rise to the exterior Dirichletto-Neumann map (or: exterior Steklov-Poincaré operator)

$$
\mathfrak{S}:=\mathfrak{W}+\left(\mathfrak{K}^{\prime}-\frac{1}{2}\right) \mathfrak{V}^{-1}\left(\mathfrak{K}-\frac{1}{2}\right) \in L\left(H^{1 / 2}(\Gamma) ; H^{-1 / 2}(\Gamma)\right)
$$

which is elliptic, i.e, $\langle\mathfrak{S} v, v\rangle \gtrsim\|v\|_{H^{1 / 2}(\Gamma)}^{2}$ for all $v \in H^{1 / 2}(\Gamma)$, see [13], Lemma 3.4, and Appendix A below. Recall that $\widetilde{H}^{-1}(\Omega)$ is the dual space of $H^{1}(\Omega)$ with respect to the $L^{2}(\Omega)$-scalar product. We define the (in general nonlinear) operator

$$
\mathfrak{A}: H^{1}(\Omega) \rightarrow \widetilde{H}^{-1}(\Omega) \quad \text { by } \quad \mathfrak{A}(u)[v]:=\langle A \nabla u, \nabla v\rangle_{\Omega}+\langle\mathfrak{S} u, v\rangle_{\Gamma}
$$

and the right-hand side

$$
L \in \widetilde{H}^{-1}(\Omega) \quad \text { by } \quad L v:=\langle f, v\rangle_{\Omega}+\left\langle\phi_{0}+\mathfrak{W} u_{0}, v\right\rangle_{\Gamma} .
$$

Then, (2.3) is equivalently recast into the operator equation

$$
\mathfrak{A}(u)=L \quad \text { in } \widetilde{H}^{-1}(\Omega) .
$$

The operator $\mathfrak{A}$ is Lipschitz continuous and strongly monotone, i.e.

$$
\|\mathfrak{A} u-\mathfrak{A} v\|_{\widetilde{H}^{-1}(\Omega)} \lesssim\|u-v\|_{H^{1}(\Omega)} \quad \text { as well as } \quad\|u-v\|_{H^{1}(\Omega)}^{2} \lesssim\langle\mathcal{A} u-\mathfrak{A} v, u-v\rangle_{\Omega} .
$$

Consequently, the main theorem on strongly monotone operators [33], Section 25.4, proves that (2.12) and thus (2.3) have a unique solution.

\subsection{Galerkin discretization}

Let $\mathcal{T}_{\ell}$ be a regular triangulation of $\Omega$ into compact triangles $T_{j} \in \mathcal{T}_{\ell}$ and $\mathcal{E}_{\ell}$ be a partition of the coupling boundary $\Gamma$ into compact and piecewise affine line segments $E_{j} \in \mathcal{E}_{\ell}$. Since $\Omega$ is polygonal, we assume that $\Omega$ as well as $\Gamma$ are exactly resolved by $\mathcal{T}_{\ell}$ and $\mathcal{E}_{\ell}$. Let $\operatorname{diam}(\omega)$ denote the Euclidean diameter of a set $\omega \subset \mathbb{R}^{2}$. For $x \in \tau \in \mathcal{T}_{\ell} \cup \mathcal{E}_{\ell}$, we define the local mesh-width function by $h_{\ell}(x):=\operatorname{diam}(\tau)$. 
For the discretization, we use a conforming discretization with continuous and $\mathcal{T}_{\ell}$-piecewise affine finite elements in $\Omega$ and $\mathcal{E}_{\ell}$-piecewise constants on $\Gamma$, i.e. the discrete spaces read

$$
\mathcal{X}_{\ell}:=\mathcal{S}^{1}\left(\mathcal{T}_{\ell}\right) \times \mathcal{P}^{0}\left(\mathcal{E}_{\ell}\right) \subseteq H^{1}(\Omega) \times H^{-1 / 2}(\Gamma)=\mathcal{H}
$$

We stress that the analysis does not enforce any coupling of $\mathcal{E}_{\ell}$ and $\mathcal{T}_{\ell}$. In particular, we do not need to assume that the boundary mesh $\mathcal{E}_{\ell}$ coincides with the restriction $\left.\mathcal{T}_{\ell}\right|_{\Gamma}$. However, for the ease of implementation, we will consider $\mathcal{E}_{\ell}=\left.\mathcal{T}_{\ell}\right|_{\Gamma}$ in the numerical experiments of Section 5 .

The Galerkin formulation of $(2.3)$ then reads as follows: find $\mathbf{U}_{\ell}^{\star}=\left(U_{\ell}^{\star}, \Phi_{\ell}^{\star}\right) \in \mathcal{X}_{\ell}$ such that

$$
\left\{\begin{aligned}
\left\langle A \nabla U_{\ell}^{\star}, \nabla V_{\ell}\right\rangle_{\Omega}+\left\langle\mathfrak{W} U_{\ell}^{\star}+\left(\mathfrak{K}^{\prime}-\frac{1}{2}\right) \Phi_{\ell}^{\star}, V_{\ell}\right\rangle_{\Gamma} & =\left\langle f, V_{\ell}\right\rangle_{\Omega}+\left\langle\phi_{0}+\mathfrak{W} u_{0}, V_{\ell}\right\rangle_{\Gamma}, \\
\left\langle\Psi_{\ell}, \mathfrak{V} \Phi_{\ell}^{\star}-\left(\mathfrak{K}-\frac{1}{2}\right) U_{\ell}^{\star}\right\rangle_{\Gamma} & =-\left\langle\Psi_{\ell},\left(\mathfrak{K}-\frac{1}{2}\right) u_{0}\right\rangle_{\Gamma},
\end{aligned}\right.
$$

for all $\mathbf{V}_{\ell}=\left(V_{\ell}, \Psi_{\ell}\right) \in \mathcal{X}_{\ell}$.

To proof the unique solvability of (2.14), the analysis of [13], Section 3, mimics the proof of the continuous case. Namely, (2.14) is equivalently rewritten in terms of a (in general nonlinear) operator equation

$$
\mathfrak{A}_{\ell}\left(U_{\ell}\right)=L_{\ell} \quad \text { in } \mathcal{S}^{1}\left(\mathcal{T}_{\ell}\right)^{*},
$$

where the nonlinear operator $\mathfrak{A}_{\ell}$ reads

$$
\mathfrak{A}_{\ell}: \mathcal{S}^{1}\left(\mathcal{T}_{\ell}\right) \rightarrow \mathcal{S}^{1}\left(\mathcal{T}_{\ell}\right)^{*}, \quad \mathfrak{A}_{\ell}\left(U_{\ell}\right)\left[V_{\ell}\right]:=\left\langle A \nabla U_{\ell}, \nabla V_{\ell}\right\rangle_{\Omega}+\left\langle\mathfrak{S}_{\ell} U_{\ell}, V_{\ell}\right\rangle_{\Gamma},
$$

cf. [13], Corollary 3. Here, $\mathfrak{S}_{\ell}$ denotes a discrete Dirichlet-to-Neumann map, which arises from the elimination of $\Phi_{\ell}^{\star}$ in the first equation of (2.14). It can be shown that $\mathfrak{S}_{\ell}$ is uniformly elliptic, where the constant depends only on $\Omega$ but not on $\mathcal{X}_{\ell}$, see Appendix A. As in the continuous case, this implies that the Lipschitz continuous operator $\mathfrak{A}_{\ell}$ is strongly monotone. Again, the main theorem on strongly monotone operators [33], Section 25.4, proves the unique solvability of (2.15) and thus of (2.14).

Moreover, uniform ellipticity of $\mathfrak{S}_{\ell}$ implies that the unique discrete solution $\mathbf{U}_{\ell}^{\star} \in \mathcal{X}_{\ell}$ is quasi optimal in the sense of the Céa lemma

$$
\left\|\mathbf{u}-\mathbf{U}_{\ell}^{\star}\right\| \leq C_{1} \min _{\mathbf{V}_{\ell} \in \mathcal{X}_{\ell}}\left\|\mathbf{u}-\mathbf{V}_{\ell}\right\|
$$

where the constant $C_{1}>0$ depends only on $\Omega$, see [13], Corollary 3, resp. [33], Corollary 25.7. Here, the natural energy norm on the energy space $\mathcal{H}$ is given by

$$
\|\mathbf{v}\|=\left(\|v\|_{H^{1}(\Omega)}^{2}+\langle\psi, \mathfrak{V} \psi\rangle_{\Gamma}\right)^{1 / 2} \quad \text { for } \mathbf{v}:=(v, \psi) \in \mathcal{H}
$$

We stress that $\mathcal{H}$ associated with $\|\cdot\| \|$ is a Hilbert space, since $\|\psi\|_{\mathfrak{V}}:=\langle\psi, \mathfrak{V} \psi\rangle_{\Gamma}^{1 / 2}$ defines an equivalent norm on $H^{-1 / 2}(\Gamma)$.

\subsection{Perturbed Galerkin discretization}

The right-hand side of the discrete formulation (2.14) involves the evaluation of $\mathfrak{W} u_{0}$ and $\mathfrak{K} u_{0}$, which can hardly be performed analytically. Moreover, so-called fast methods for boundary integral operators usually deal with discrete functions, $c f$. [29]. Therefore, we propose to approximate at least the given boundary data $u_{0} \in$ $H^{1 / 2}(\Gamma)$ by appropriate discrete functions and proceed analogously to [2]: to that end and to provide below a local measure for the approximation error, we assume additional regularity $u_{0} \in H^{1}(\Gamma)$. According to the Sobolev inequality in $1 \mathrm{D}, u_{0}$ is continuous. Therefore, we may consider the nodal interpolant

$$
U_{0, \ell}:=I_{\ell}^{\Gamma} u_{0}=\sum_{j=1}^{n} u_{0}\left(z_{j}\right) \zeta_{j} \in \mathcal{S}^{1}\left(\mathcal{E}_{\ell}\right)
$$


where $z_{j} \in \Gamma$ denotes a node of $\mathcal{E}_{\ell}$ and where $\zeta_{j}$ is the associated $\mathcal{E}_{\ell}$-piecewise linear and continuous hat function, i.e., $\zeta_{j}\left(z_{k}\right)=\delta_{j k}$. Now, the perturbed Galerkin formulation reads as follows: Find $\mathbf{U}_{\ell}=\left(U_{\ell}, \Phi_{\ell}\right) \in \mathcal{X}_{\ell}$ such that

$$
\left\{\begin{aligned}
\left\langle A \nabla U_{\ell}, \nabla V_{\ell}\right\rangle_{\Omega}+\left\langle\mathfrak{W} U_{\ell}+\left(\mathfrak{K}^{\prime}-\frac{1}{2}\right) \Phi_{\ell}, V_{\ell}\right\rangle_{\Gamma} & =\left\langle f, V_{\ell}\right\rangle_{\Omega}+\left\langle\phi_{0}+\mathfrak{W} U_{0, \ell}, V_{\ell}\right\rangle_{\Gamma}, \\
\left\langle\Psi_{\ell}, \mathfrak{V} \Phi_{\ell}-\left(\mathfrak{K}-\frac{1}{2}\right) U_{\ell}\right\rangle_{\Gamma} & =-\left\langle\Psi_{\ell},\left(\mathfrak{K}-\frac{1}{2}\right) U_{0, \ell}\right\rangle_{\Gamma},
\end{aligned}\right.
$$

for all $\mathbf{V}_{\ell}=\left(V_{\ell}, \Psi_{\ell}\right) \in \mathcal{X}_{\ell}$. Compared to (2.14), the only difference is that (2.20) involves the approximate data $U_{0, \ell}$ instead of $u_{0}$ on the right-hand side. Consequently, the same arguments as before prove that (2.20) has a unique solution.

\section{A POSTERIORI ERROR ESTIMATION}

\subsection{Local mesh-refinement}

For the local refinement of the volume mesh $\mathcal{T}_{\ell}$, we use newest vertex bisection, where marked triangles $T \in \mathcal{T}_{\ell}$ are refined by $\operatorname{bisec}_{3}(T)$. We refer to [32], Chapter 5 , for details on newest vertex bisection. The mesh $\widehat{\mathcal{T}}_{\ell}$ is obtained from uniform bisec $_{3}$-refinement of $\mathcal{T}_{\ell}$. This ensures uniform shape regularity of the triangulations $\mathcal{T}_{\ell}$ and $\widehat{\mathcal{T}}_{\ell}$. More precisely, the shape regularity constant

$$
\sigma\left(\mathcal{T}_{\ell}\right):=\max \left\{\operatorname{diam}(T)^{2} /|T|: T \in \mathcal{T}_{\ell}\right\}
$$

depends only on the initial mesh $\mathcal{T}_{0}$, i.e.

$$
\max \left\{\sup _{\ell \in \mathbb{N}} \sigma\left(\mathcal{T}_{\ell}\right), \sup _{\ell \in \mathbb{N}} \sigma\left(\widehat{\mathcal{T}}_{\ell}\right)\right\} \leq C \sigma\left(\mathcal{T}_{0}\right),
$$

where $C>$ depends only on the labelling of the reference edges in $\mathcal{T}_{0}$. Furthermore, there holds nestedness of the associated spaces

$$
\mathcal{S}^{1}\left(\mathcal{T}_{\ell}\right) \subseteq \mathcal{S}^{1}\left(\mathcal{T}_{\ell+1}\right) \subseteq \mathcal{S}^{1}\left(\widehat{\mathcal{T}}_{\ell}\right) \subseteq \mathcal{S}^{1}\left(\widehat{\mathcal{T}}_{\ell+1}\right)
$$

For the local refinement of the boundary mesh $\mathcal{E}_{\ell}$, we use bisection of the marked elements, i.e. marked elements $E \in \mathcal{E}_{\ell}$ are refined into two son elements with halved diameter. Since the error estimates below depend on the local mesh-ratio (also called $K$-mesh constant)

$$
\kappa\left(\mathcal{E}_{\ell}\right):=\max \left\{\operatorname{diam}(E) / \operatorname{diam}\left(E^{\prime}\right): E, E^{\prime} \in \mathcal{E}_{\ell} \text { with } E \cap E^{\prime} \neq \emptyset\right\},
$$

one has to do some additional marking to ensure

$$
\sup _{T \in \mathcal{T}_{\ell}} \kappa\left(\mathcal{E}_{\ell}\right) \leq 2 \kappa\left(\mathcal{E}_{0}\right)
$$

cf. [2], Section 2.2, for details. The mesh $\widehat{\mathcal{E}}_{\ell}$ is obtained from uniform refinement of $\mathcal{E}_{\ell}$, whence $\kappa\left(\mathcal{E}_{\ell}\right)=\kappa\left(\widehat{\mathcal{E}}_{\ell}\right)$.

Alternatively, one may consider the boundary partition $\mathcal{E}_{\ell}:=\left.\mathcal{T}_{\ell}\right|_{\Gamma}$ induced by the triangulation $\mathcal{T}_{\ell}$ of $\Omega$. Then, marking of an element $E \in \mathcal{E}_{\ell}$ means marking of certain edges of some triangles $T \in \mathcal{T}_{\ell}$ for newest vertex bisection. We stress that this also guarantees that marked elements $E$ are split into two son elements of half length. Moreover, due to uniform shape regularity (3.2) of $\mathcal{T}_{\ell}$, there automatically holds

$$
\sup _{T \in \mathcal{T}_{\ell}} \kappa\left(\mathcal{E}_{\ell}\right) \leq C \kappa\left(\mathcal{E}_{0}\right)
$$

with some constant $C>0$ which depends only on $\mathcal{T}_{0}$. 
In any case, there again holds nestedness

$$
\mathcal{P}^{0}\left(\mathcal{T}_{\ell}\right) \subseteq \mathcal{P}^{0}\left(\mathcal{T}_{\ell+1}\right) \subseteq \mathcal{P}^{0}\left(\widehat{\mathcal{T}}_{\ell}\right) \subseteq \mathcal{P}^{0}\left(\widehat{\mathcal{T}}_{\ell+1}\right)
$$

Finally, we consider the discrete spaces $\mathcal{X}_{\ell}:=\mathcal{S}^{1}\left(\mathcal{T}_{\ell}\right) \times \mathcal{P}^{0}\left(\mathcal{E}_{\ell}\right)$ as well as $\widehat{\mathcal{X}}_{\ell}:=\mathcal{S}^{1}\left(\widehat{\mathcal{T}}_{\ell}\right) \times \mathcal{P}^{0}\left(\widehat{\mathcal{E}}_{\ell}\right)$. We note that

$$
\mathcal{X}_{\ell} \subseteq \mathcal{X}_{\ell+1} \subseteq \widehat{\mathcal{X}}_{\ell} \subseteq \widehat{\mathcal{X}}_{\ell+1} \subset \mathcal{H}
$$

where only the inclusions $\mathcal{X}_{\ell} \subseteq \mathcal{X}_{\ell+1}, \mathcal{X}_{\ell} \subseteq \widehat{\mathcal{X}}_{\ell}$, and $\widehat{\mathcal{X}}_{\ell} \subseteq \widehat{\mathcal{X}}_{\ell+1}$ are mandatory for the analysis below.

\subsection{A posteriori error control for data approximation}

Instead of solving the non-perturbed Galerkin formulation (2.14) of the weak formulation (2.3), we solve the perturbed Galerkin formulation (2.20) in practice. Put differently, $\mathbf{U}_{\ell}=\left(U_{\ell}, \Phi_{\ell}\right) \in \mathcal{X}_{\ell}$ is the Galerkin approximation of the unique solution $\mathbf{u}_{\ell}=\left(u_{\ell}, \phi_{\ell}\right) \in \mathcal{H}$ of the perturbed formulation

$$
\left\{\begin{array}{l}
\left\langle A \nabla u_{\ell}, \nabla v\right\rangle_{\Omega}+\left\langle\mathfrak{W} u_{\ell}+\left(\mathfrak{K}^{\prime}-\frac{1}{2}\right) \phi_{\ell}, v\right\rangle_{\Gamma}=\langle f, v\rangle_{\Omega}+\left\langle\phi_{0}+\mathfrak{W} U_{0, \ell}, v\right\rangle_{\Gamma}, \\
\left\langle\psi, \mathfrak{V} \phi_{\ell}-\left(\mathfrak{K}-\frac{1}{2}\right) u_{\ell}\right\rangle_{\Gamma}=-\left\langle\psi,\left(\mathfrak{K}-\frac{1}{2}\right) U_{0, \ell}\right\rangle_{\Gamma},
\end{array}\right.
$$

for all $\mathbf{v}=(v, \psi) \in \mathcal{H}$. In this section, we aim to verify a computable upper bound to control the approximation errors $\left\|\mathbf{u}-\mathbf{u}_{\ell}\right\|$ and $\left\|\mathbf{U}_{\ell}^{\star}-\mathbf{U}_{\ell}\right\|$, stated in the following proposition.

Proposition 3.1. There holds the approximation error estimate

$$
C_{2}^{-1}\left\|\mathbf{U}_{\ell}^{\star}-\mathbf{U}_{\ell}\right\| \leq\left\|\mathbf{u}-\mathbf{u}_{\ell}\right\| \leq C_{3} \operatorname{osc}_{\ell},
$$

where

$$
\operatorname{osc}_{\ell}:=\left\|h_{\ell}^{1 / 2}\left(u_{0}-U_{0, \ell}\right)^{\prime}\right\|_{L^{2}(\Gamma)} .
$$

Here, $(\cdot)^{\prime}$ denotes the arclength derivative along $\Gamma$. The constant $C_{2}>0$ depends only on $\Omega$, whereas $C_{3}>0$ additionally depends on the $K$-mesh constant $\kappa\left(\mathcal{E}_{0}\right)$.

The proof of (3.7) is essentially based on the following stability result.

Lemma 3.2. Let $\mathbf{u}=(u, \phi) \in \mathcal{H}$ be the exact solution of (2.3) for given data $\left(f, u_{0}, \phi_{0}\right) \in \mathcal{D}:=\widetilde{H}^{-1}(\Omega) \times$ $H^{1 / 2}(\Gamma) \times H^{-1 / 2}(\Gamma)$. Let $\widetilde{\mathbf{u}}=(\widetilde{u}, \widetilde{\phi}) \in \mathcal{H}$ be the exact solution of $(2.3)$ for given data $\left(\widetilde{f}, \widetilde{u}_{0}, \widetilde{\phi}_{0}\right) \in \mathcal{D}$. Let $\mathbf{U}_{\ell}^{\star}, \widetilde{\mathbf{U}}_{\ell}^{\star} \in \mathcal{X}_{\ell}$ be the respective Galerkin solutions of (2.14). Then,

$$
C_{2}^{-1}\left\|\mathbf{U}_{\ell}^{\star}-\widetilde{\mathbf{U}}_{\ell}^{\star}\right\| \leq\|\mathbf{u}-\widetilde{\mathbf{u}}\| \leq C_{4}\left(\|f-\widetilde{f}\|_{\widetilde{H}^{-1}(\Omega)}+\left\|u_{0}-\widetilde{u}_{0}\right\|_{H^{1 / 2}(\Gamma)}+\left\|\phi_{0}-\widetilde{\phi}_{0}\right\|_{H^{-1 / 2}(\Gamma)}\right),
$$

where the constants $C_{2}, C_{4}>0$ depend only on $\Omega$.

Proof. We proceed as above in Section 2.1 to rewrite the weak formulations (2.3) for $\mathbf{u}$ and $\widetilde{\mathbf{u}}$. Note that the second equations of both formulations are equivalently written as

$$
\phi=\mathfrak{V}^{-1}\left(\mathfrak{K}-\frac{1}{2}\right)\left(u-u_{0}\right) \text { as well as } \widetilde{\phi}=\mathfrak{V}^{-1}\left(\mathfrak{K}-\frac{1}{2}\right)\left(\widetilde{u}-\widetilde{u}_{0}\right) .
$$

With the operator $\mathfrak{A}$ from $(2.10)$ and the right-hand sides $L(\cdot) \in \widetilde{H}^{-1}(\Omega)$ defined by

$$
L\left(f, u_{0}, \phi_{0}\right)[v]:=\langle f, v\rangle_{\Omega}+\left\langle\phi_{0}+\mathfrak{W} u_{0}, v\right\rangle_{\Gamma},
$$


the variational formulations (2.3) are equivalently recast into the operator equations

$$
\mathfrak{A}(u)=L\left(f, u_{0}, \phi_{0}\right) \quad \text { resp. } \mathfrak{A}(\widetilde{u})=L\left(\widetilde{f}, \widetilde{u}_{0}, \widetilde{\phi}_{0}\right) .
$$

As mentioned above, $\mathfrak{A}$ is Lipschitz continuous and strongly monotone, whence bijective. Moreover, the inverse of a strongly monotone operator is Lipschitz continuous since $\|u-v\|_{H^{1}(\Omega)} \lesssim\|\mathfrak{A} u-\mathfrak{A} v\|_{\widetilde{H}^{-1}(\Omega)}$. This and the continuity of $\mathfrak{S}$ imply

$$
\begin{aligned}
\|u-\widetilde{u}\|_{H^{1}(\Omega)} & \lesssim\left\|L\left(f, u_{0}, \phi_{0}\right)-L\left(\widetilde{f}, \widetilde{u}_{0}, \widetilde{\phi}_{0}\right)\right\|_{\widetilde{H}^{-1}(\Omega)} \\
& \leq\|f-\widetilde{f}\|_{\widetilde{H}^{-1}(\Omega)}+\left\|\phi_{0}-\widetilde{\phi}_{0}\right\|_{H^{-1 / 2}(\Gamma)}+\left\|\mathfrak{S}\left(u_{0}-\widetilde{u}_{0}\right)\right\|_{H^{-1 / 2}(\Gamma)} \\
& \lesssim\|f-\widetilde{f}\|_{\widetilde{H}^{-1}(\Omega)}+\left\|\phi_{0}-\widetilde{\phi}_{0}\right\|_{H^{-1 / 2}(\Gamma)}+\left\|u_{0}-\widetilde{u}_{0}\right\|_{H^{1 / 2}(\Gamma)} .
\end{aligned}
$$

Next, the mapping properties of the integral operators $\mathfrak{V}$ and $\mathfrak{K}$ yield

$$
\begin{aligned}
\|\phi-\widetilde{\phi}\|_{H^{-1 / 2}(\Gamma)} & =\left\|\mathfrak{V}^{-1}\left(\mathfrak{K}-\frac{1}{2}\right)\left((u-\widetilde{u})-\left(u_{0}-\widetilde{u}_{0}\right)\right)\right\|_{H^{-1 / 2}(\Gamma)} \\
& \lesssim\|u-\widetilde{u}\|_{H^{1 / 2}(\Gamma)}+\left\|u_{0}-\widetilde{u}_{0}\right\|_{H^{1 / 2}(\Gamma)} .
\end{aligned}
$$

Combining the latter two estimates and $\|u-\widetilde{u}\|_{H^{1 / 2}(\Gamma)} \lesssim\|u-\widetilde{u}\|_{H^{1}(\Omega)}$, we obtain

$$
\|\mathbf{u}-\widetilde{\mathbf{u}}\| \lesssim\|f-\widetilde{f}\|_{\widetilde{H}^{-1}(\Omega)}+\left\|\phi_{0}-\widetilde{\phi}_{0}\right\|_{H^{-1 / 2}(\Gamma)}+\left\|u_{0}-\widetilde{u}_{0}\right\|_{H^{1 / 2}(\Gamma)} .
$$

This proves the upper estimate in (3.9).

To estimate $\left\|\mathbf{U}_{\ell}^{\star}-\widetilde{\mathbf{U}}_{\ell}^{\star}\right\|$, we use the same type of arguments with the discrete operator $\mathfrak{A}_{\ell}$. For instance, we rewrite $(2.14)$ as

$$
\left\{\begin{array}{l}
\left\langle A \nabla U_{\ell}^{\star}, \nabla V_{\ell}\right\rangle_{\Omega}+\left\langle\mathfrak{W} U_{\ell}^{\star}+\left(\mathfrak{K}^{\prime}-\frac{1}{2}\right) \Phi_{\ell}^{\star}, V_{\ell}\right\rangle_{\Gamma}=\left\langle A \nabla u, \nabla V_{\ell}\right\rangle_{\Omega}+\left\langle\mathfrak{W} u+\left(\mathfrak{K}^{\prime}-\frac{1}{2}\right) \phi, V_{\ell}\right\rangle_{\Gamma}, \\
\left\langle\Psi_{\ell}, \mathfrak{V} \Phi_{\ell}^{\star}-\left(\mathfrak{K}-\frac{1}{2}\right) U_{\ell}^{\star}\right\rangle_{\Gamma}=\left\langle\Psi_{\ell}, \mathfrak{V} \phi-\left(\mathfrak{K}-\frac{1}{2}\right) u\right\rangle_{\Gamma},
\end{array}\right.
$$

for all $\mathbf{V}_{\ell}=\left(V_{\ell}, \Phi_{\ell}\right) \in \mathcal{X}_{\ell}$. With the discrete Steklov-Poincaré operator $\mathfrak{S}_{\ell}$ and the operator $\mathfrak{A}_{\ell}$, this becomes

$$
\mathfrak{A}_{\ell} U_{\ell}^{\star}=L_{\ell}(u, \phi)
$$

with a certain right-hand side $L_{\ell}(u, \phi) \in \mathcal{S}^{1}\left(\mathcal{T}_{\ell}\right)^{*}$. Replacing $\mathbf{u}=(u, \phi)$ by $\widetilde{\mathbf{u}}=(\widetilde{u}, \widetilde{\phi})$, we obtain the discrete operator formulation for $\widetilde{U}_{\ell}^{\star}$. It is easily seen that Lipschitz continuity of the inverse of $\mathfrak{A}_{\ell}$ thus yields

$$
\left\|U_{\ell}^{\star}-\widetilde{U}_{\ell}^{\star}\right\|_{H^{1}(\Omega)} \lesssim\left\|L_{\ell}(u, \phi)-L_{\ell}(\widetilde{u}, \widetilde{\phi})\right\|_{\mathcal{S}^{1}\left(\mathcal{T}_{\ell}\right)^{*}} \lesssim\|\mathbf{u}-\widetilde{\mathbf{u}}\| .
$$

Using the discrete simple-layer potential, one additionally obtains

$$
\left\|\Phi_{\ell}^{\star}-\widetilde{\Phi}_{\ell}^{\star}\right\|_{H^{-1 / 2}(\Gamma)} \lesssim\left\|U_{\ell}^{\star}-\widetilde{U}_{\ell}^{\star}\right\|_{H^{1}(\Omega)}+\|\mathbf{u}-\widetilde{\mathbf{u}}\| \lesssim\|\mathbf{u}-\widetilde{\mathbf{u}}\| .
$$

Altogether, we thus see $\left\|\mathbf{U}_{\ell}^{\star}-\widetilde{\mathbf{U}}_{\ell}^{\star}\right\| \lesssim\|\mathbf{u}-\widetilde{\mathbf{u}}\|$ and conclude the proof.

The a posteriori error control of the approximation of $u_{0}$ by $U_{0, \ell}$ is now done via an approximation result from [8], Theorem 1. Our formulation in equation (3.10), taken from [19], Lemma 2.2, is a consequence of the latter. 
Lemma 3.3. With $I_{\ell}^{\Gamma}: H^{1}(\Gamma) \rightarrow \mathcal{S}^{1}\left(\mathcal{E}_{\ell}\right)$ the nodal interpolation operator and $\Pi_{\ell}^{\Gamma}: L^{2}(\Gamma) \rightarrow \mathcal{P}^{0}\left(\mathcal{E}_{\ell}\right)$ the $L^{2}$ orthogonal projection, there holds $\Pi_{\ell}^{\Gamma}\left(v^{\prime}\right)=\left(I_{\ell}^{\Gamma} v\right)^{\prime}$, where $(\cdot)^{\prime}$ denotes the arclength derivative. Moreover, there holds the approximation result

$$
C_{5}^{-1}\left\|v-I_{\ell}^{\Gamma} v\right\|_{H^{1 / 2}(\Gamma)} \leq\left\|h_{\ell}^{1 / 2}\left(v-I_{\ell}^{\Gamma} v\right)^{\prime}\right\|_{L^{2}(\Gamma)} \leq\left\|h_{\ell}^{1 / 2} v^{\prime}\right\|_{L^{2}(\Gamma)} \quad \text { for all } v \in H^{1}(\Gamma),
$$

and the constant $C_{5}>0$ depends only on $\Gamma$ and $\kappa\left(\mathcal{E}_{0}\right)$.

Proof of Proposition 3.1. According to Lemma 3.2, there holds

$$
\left\|\mathbf{U}_{\ell}^{\star}-\mathbf{U}_{\ell}\right\| \lesssim\left\|\mathbf{u}-\mathbf{u}_{\ell}\right\| \lesssim\left\|u_{0}-U_{0, \ell}\right\|_{H^{1 / 2}(\Gamma)} .
$$

By choice of the discrete approximation $U_{0, \ell}=I_{\ell}^{\Gamma} u_{0}$, the approximation estimate (3.10) yields

$$
\left\|u_{0}-U_{0, \ell}\right\|_{H^{1 / 2}(\Gamma)} \lesssim\left\|h_{\ell}^{1 / 2}\left(u_{0}-U_{0, \ell}\right)^{\prime}\right\|_{L^{2}(\Gamma)} .
$$

This concludes the proof.

\subsection{Saturation assumption and $(h-h / 2)$-error estimator}

Let $\widehat{\mathcal{T}}_{\ell}$ and $\widehat{\mathcal{E}}_{\ell}$ be the uniform refinements of $\mathcal{T}_{\ell}$ and $\mathcal{E}_{\ell}$, respectively, and define

$$
\widehat{\mathcal{X}}_{\ell}=\mathcal{S}^{1}\left(\widehat{\mathcal{T}}_{\ell}\right) \times \mathcal{P}^{0}\left(\widehat{\mathcal{E}}_{\ell}\right) .
$$

In this section, we consider the canonical $(h-h / 2)$-error estimators

$$
\eta_{\ell}^{\star}:=\left\|\widehat{\mathbf{U}}_{\ell}^{\star}-\mathbf{U}_{\ell}^{\star}\right\| \quad \text { and } \quad \eta_{\ell}:=\left\|\widehat{\mathbf{U}}_{\ell}-\mathbf{U}_{\ell}\right\| .
$$

Here, $\widehat{\mathbf{U}}_{\ell}^{\star}, \widehat{\mathbf{U}}_{\ell} \in \widehat{\mathcal{X}}_{\ell}$ are the Galerkin solutions of (2.14) and (2.20) with respect to the uniformly refined meshes. We remark that due to the non-locality of $\|\cdot\|_{\mathfrak{V}}$, the error estimator $\eta_{\ell}$ does not provide any information where to refine the boundary partition $\mathcal{E}_{\ell}$. This will be different for the error estimators considered in the subsequent section.

Lemma 3.4. There is a constant $C_{6}>0$ which depends only on $\Omega$ such that

$$
\eta_{\ell}^{\star} \leq C_{6}\left\|\mathbf{u}-\mathbf{U}_{\ell}^{\star}\right\| .
$$

Under the so-called saturation assumption

$$
\left\|\mathbf{u}-\widehat{\mathbf{U}}_{\ell}^{\star}\right\| \leq C_{\text {sat }}\left\|\mathbf{u}-\mathbf{U}_{\ell}^{\star}\right\|
$$

with some $\ell$-independent constant $0<C_{\text {sat }}<1$, there holds

$$
\left\|\mathbf{u}-\mathbf{U}_{\ell}^{\star}\right\| \leq \frac{1}{1-C_{\mathrm{sat}}} \eta_{\ell}^{\star} .
$$

Proof. To prove (3.12), we use $\mathbf{U}_{\ell}^{\star} \in \mathcal{X}_{\ell} \subseteq \widehat{\mathcal{X}}_{\ell}$ and the quasi-optimality (2.17) applied for $\widehat{\mathbf{U}}_{\ell}^{\star} \in \widehat{\mathcal{X}}_{\ell}$. This and the triangle inequality yield

$$
\eta_{\ell}^{\star} \leq\left\|\mathbf{u}-\widehat{\mathbf{U}}_{\ell}^{\star}\right\|+\left\|\mathbf{u}-\mathbf{U}_{\ell}^{\star}\right\| \lesssim\left\|\mathbf{u}-\mathbf{U}_{\ell}^{\star}\right\| .
$$

For the converse inequality, the triangle inequality and the saturation assumption (3.13) provide

$$
\left\|\mathbf{u}-\mathbf{U}_{\ell}^{\star}\right\| \leq\left\|\mathbf{u}-\widehat{\mathbf{U}}_{\ell}^{\star}\right\|+\eta_{\ell}^{\star} \leq C_{\text {sat }}\left\|\mathbf{u}-\mathbf{U}_{\ell}^{\star}\right\| \|+\eta_{\ell}^{\star} .
$$

Rearranging the terms, we conclude (3.14). 


\section{Remark 3.5.}

(a) We remark that the saturation assumption (3.13) dates back to the early work [6], but may fail to hold in general $[7,17]$. However, it essentially states that the numerical scheme has reached an asymptotic phase [20], Section 5.2;

(b) for model problems and lowest-order FEM, the saturation assumption (3.13) can be proven, if the given data are sufficiently resolved. More precisely, [17] then states

$$
\left\|\mathbf{u}-\widehat{\mathbf{U}}_{\ell}^{\star}\right\| \leq C_{\text {sat }}\left\|\mathbf{u}-\mathbf{U}_{\ell}^{\star}\right\|+\operatorname{osc}_{\ell},
$$

so that small data oscillation implies the saturation assumption. This led to the incorporation of the data resolution into the convergent $(h-h / 2)$-estimator steered adaptive algorithm of [21], where $\eta_{\ell}+$ osc $_{\ell}$ is used to drive the adaptive FE algorithm. One may expect that a result similar to that of [17] should also hold for BEM or the FEM-BEM coupling. However, the non-locality of the involved boundary integral operators imposes severe difficulties, and we expect that new mathematical techniques have to be developed;

(c) the results of $[17,21]$ mentioned before in (b) provide an additional reason why one should include the resolution of the given data into the adaptive scheme and may consider discretized data $U_{0, \ell}$ of $u_{0}$. The inclusion of data approximation terms for the approximation of $\phi_{0}$ and $f$ into our analysis is easily possible, but neglected for the ease of presentation;

(d) formally, we stress that the saturation assumption (3.13) is not used for any arbitrary mesh $\mathcal{T}_{\ell}$, but only for the sequence of meshes which are generated by the adaptive algorithm below, see Section 4.3. This might be one reason why the saturation assumption (3.13) is usually observed in numerical experiments [20,21].

Lemma 3.6. There is a constant $C_{7}>0$ which depends only on $\Omega$ and $\kappa\left(\mathcal{E}_{0}\right)$, such that

$$
\eta_{\ell} \leq \eta_{\ell}^{\star}+C_{7} \operatorname{osc}_{\ell} \text { as well as } \eta_{\ell}^{\star} \leq \eta_{\ell}+C_{7} \operatorname{osc}_{\ell} \text {. }
$$

Proof. The triangle inequality and Proposition 3.1 prove

$$
\eta_{\ell} \leq \eta_{\ell}^{\star}+\left\|\widehat{\mathbf{U}}_{\ell}^{\star}-\widehat{\mathbf{U}}_{\ell}\right\|+\left\|\mathbf{U}_{\ell}^{\star}-\mathbf{U}_{\ell}\right\| \leq \eta_{\ell}^{\star}+2 C_{3} \operatorname{osc}_{\ell} .
$$

The converse inequality follows along the same lines.

Proposition 3.7. There is a constant $C_{8}>0$ which depends only on $\Omega$ and $\kappa\left(\mathcal{E}_{0}\right)$ such that

$$
C_{8}^{-1} \eta_{\ell} \leq\left\|\mathbf{u}-\mathbf{U}_{\ell}\right\|+\operatorname{osc}_{\ell} .
$$

Under the saturation assumption (3.13), there is a constant $C_{9}>0$ such that

$$
C_{9}^{-1}\left\|\mathbf{u}-\mathbf{U}_{\ell}\right\| \leq \eta_{\ell}+\operatorname{osc}_{\ell} .
$$

Besides $\Omega$ and $\kappa\left(\mathcal{E}_{0}\right)$, the constant $C_{9}>0$ depends only on $0<C_{\text {sat }}<1$.

Proof. The proof is a consequence of Lemmas 3.4 and 3.6. For instance, there holds

$$
\eta_{\ell} \lesssim \eta_{\ell}^{\star}+\operatorname{osc}_{\ell} \lesssim\left\|\mathbf{u}-\mathbf{U}_{\ell}^{\star}\right\|+\operatorname{osc}_{\ell} \lesssim\left\|\mathbf{u}-\mathbf{U}_{\ell}\right\|+\left\|\mathbf{U}_{\ell}-\mathbf{U}_{\ell}^{\star}\right\|+\operatorname{osc}_{\ell} \lesssim\left\|\mathbf{u}-\mathbf{U}_{\ell}\right\|+\operatorname{osc}_{\ell} .
$$

This proves (3.16), and (3.17) follows along the same lines. 


\subsection{Further $(h-h / 2)$-type error estimators}

Let $\widehat{\mathbf{U}}_{\ell}=\left(\widehat{U}_{\ell}, \widehat{\Phi}_{\ell}\right) \in \widehat{\mathcal{X}}_{\ell}$ be the Galerkin solution of (2.20) with respect to $\widehat{\mathcal{X}}_{\ell}$. In addition to the error estimator $\eta_{\ell}$ from (3.11), we introduce two further error estimators. First,

$$
\mu_{\ell}:=\left(\left\|\nabla\left(1-I_{\ell}^{\Omega}\right) \widehat{U}_{\ell}\right\|_{L^{2}(\Omega)}^{2}+\left\|h_{\ell}^{1 / 2}\left(1-\Pi_{\ell}^{\Gamma}\right) \widehat{\Phi}_{\ell}\right\|_{L^{2}(\Gamma)}^{2}\right)^{1 / 2},
$$

where $I_{\ell}^{\Omega}: C(\bar{\Omega}) \rightarrow \mathcal{S}^{1}\left(\mathcal{T}_{\ell}\right)$ denotes the nodal interpolation operator and where $\Pi_{\ell}^{\Gamma}: L^{2}(\Gamma) \rightarrow \mathcal{P}^{0}\left(\mathcal{E}_{\ell}\right)$ denotes the $L^{2}(\Gamma)$-orthogonal projection. Second,

$$
\widetilde{\mu}_{\ell}:=\left(\left\|\left(1-\Pi_{\ell}^{\Omega}\right) \nabla \widehat{U}_{\ell}\right\|_{L^{2}(\Omega)}^{2}+\left\|h_{\ell}^{1 / 2}\left(1-\Pi_{\ell}^{\Gamma}\right) \widehat{\Phi}_{\ell}\right\|_{L^{2}(\Gamma)}^{2}\right)^{1 / 2}
$$

where $\Pi_{\ell}^{\Omega}: L^{2}(\Omega) \rightarrow \mathcal{P}^{0}\left(\mathcal{T}_{\ell}\right)$ denotes the $L^{2}(\Omega)$-orthogonal projection.

The local contributions of $\mu_{\ell}$ and $\widetilde{\mu}_{\ell}$ are denoted by

$$
\mu_{\ell}(T)^{2}=\left\|\nabla\left(1-I_{\ell}^{\Omega}\right) \widehat{U}_{\ell}\right\|_{L^{2}(T)}^{2} \quad \text { resp. } \quad \widetilde{\mu}_{\ell}(T)^{2}=\left\|\left(1-\Pi_{\ell}^{\Omega}\right) \nabla \widehat{U}_{\ell}\right\|_{L^{2}(T)}^{2}
$$

for triangles $T \in \mathcal{T}_{\ell}$ and by

$$
\mu_{\ell}(E)^{2}=\widetilde{\mu}_{\ell}(E)^{2}=\operatorname{diam}(E)\left\|\left(1-\Pi_{\ell}^{\Gamma}\right) \widehat{\Phi}_{\ell}\right\|_{L^{2}(E)}^{2}
$$

for line segments $E \in \mathcal{E}_{\ell}$. Note that this definition results in

$$
\mu_{\ell}^{2}=\sum_{T \in \mathcal{T}_{\ell}} \mu_{\ell}(T)^{2}+\sum_{E \in \mathcal{E}_{\ell}} \mu_{\ell}(E)^{2} \quad \text { and } \quad \widetilde{\mu}_{\ell}^{2}=\sum_{T \in \mathcal{T}_{\ell}} \widetilde{\mu}_{\ell}(T)^{2}+\sum_{E \in \mathcal{E}_{\ell}} \widetilde{\mu}_{\ell}(E)^{2} .
$$

The following lemma provides certain equivalences of the introduced error estimators.

Lemma 3.8. There are constants $C_{10}, C_{11}, C_{12}>0$ such that

$$
C_{10}^{-1} \mu_{\ell}(T) \leq \widetilde{\mu}_{\ell}(T) \leq \mu_{\ell}(T) \quad \text { for all } T \in \mathcal{T}_{\ell}
$$

as well as

$$
C_{12}^{-1} \widetilde{\mu}_{\ell} \leq \eta_{\ell} \leq C_{11} \mu_{\ell} \quad \text { and } \mu_{\ell} \leq C_{10} \widetilde{\mu}_{\ell}
$$

The constant $C_{10} \geq 1$ depends only on the shape regularity constant $\sigma\left(\mathcal{T}_{0}\right)$. The constant $C_{11}>0$ depends only on $\Omega$, whereas $C_{12}>0$ depends only on $\Omega$ and on $\kappa\left(\mathcal{E}_{0}\right)$.

Proof. Since the orthogonal projection $\Pi_{\ell}^{\Omega}$ is the $\mathcal{T}_{\ell}$-elementwise best approximation operator, the estimate $\tilde{\mu}_{\ell}(T) \leq \mu_{\ell}(T)$ is obvious. The converse inequality follows from a scaling argument. In particular, there holds $C_{10} \geq 1$. In view of (3.20)-(3.22), this also implies $\widetilde{\mu}_{\ell} \leq \mu_{\ell} \leq C_{10} \widetilde{\mu}_{\ell}$.

Moreover, the local $L^{2}$-best approximation properties of $\Pi_{\ell}^{\Omega}$ also proves

$$
\widetilde{\mu}_{\ell}(T)^{2} \leq\left\|\nabla\left(\widehat{U}_{\ell}-U_{\ell}\right)\right\|_{L^{2}(T)}^{2} \leq\left\|\widehat{U}_{\ell}-U_{\ell}\right\|_{H^{1}(T)}^{2} \quad \text { for all } T \in \mathcal{T}_{\ell}
$$

Using the $\mathcal{E}_{\ell}$-elementwise best approximation property of $\Pi_{\ell}^{\Gamma}$ and the local inverse estimate from [22], Theorem 3.6 , we obtain

$$
\left\|h_{\ell}^{1 / 2}\left(1-\Pi_{\ell}^{\Gamma}\right) \widehat{\Phi}_{\ell}\right\|_{L^{2}(\Gamma)} \leq\left\|h_{\ell}^{1 / 2}\left(\widehat{\Phi}_{\ell}-\Phi_{\ell}\right)\right\|_{L^{2}(\Gamma)} \lesssim\left\|\widehat{\Phi}_{\ell}-\Phi_{\ell}\right\|_{\mathfrak{V}}
$$


where the constant depends only on $\Gamma$ and an upper bound of $\kappa\left(\mathcal{E}_{\ell}\right)$. The combination of the last two inequalities proves $\tilde{\mu}_{\ell} \lesssim \eta_{\ell}$.

Finally, we aim at proving $\eta_{\ell} \lesssim \mu_{\ell}$. To that end, recall that $\widehat{\mathbf{U}}_{\ell}$ solves $(2.20)$ with $\mathcal{X}_{\ell}$ replaced by $\widehat{\mathcal{X}}_{\ell}$. This allows to rewrite $(2.20)$ in the form

$$
\left\{\begin{array}{l}
\left\langle A \nabla U_{\ell}, \nabla V_{\ell}\right\rangle_{\Omega}+\left\langle\mathfrak{W} U_{\ell}+\left(\mathfrak{K}^{\prime}-\frac{1}{2}\right) \Phi_{\ell}, V_{\ell}\right\rangle_{\Gamma}=\left\langle A \nabla \widehat{U}_{\ell}, \nabla V_{\ell}\right\rangle_{\Omega}+\left\langle\mathfrak{W} \widehat{U}_{\ell}+\left(\mathfrak{K}^{\prime}-\frac{1}{2}\right) \widehat{\Phi}_{\ell}, V_{\ell}\right\rangle_{\Gamma}, \\
\left\langle\Psi_{\ell}, \mathfrak{V} \Phi_{\ell}-\left(\mathfrak{K}-\frac{1}{2}\right) U_{\ell}\right\rangle_{\Gamma}=\left\langle\Psi_{\ell}, \mathfrak{V} \widehat{\Phi}_{\ell}-\left(\mathfrak{K}-\frac{1}{2}\right) \widehat{U}_{\ell}\right\rangle_{\Gamma}
\end{array}\right.
$$

for all $\mathbf{V}_{\ell}=\left(V_{\ell}, \Psi_{\ell}\right) \in \mathcal{X}_{\ell}$. Replacing $\mathbf{V}_{\ell} \in \mathcal{X}_{\ell}$ in this formulation by a general test function $\mathbf{v} \in \mathcal{H}$, we obtain a new variational formulation. By definition, $\widehat{\mathbf{U}}_{\ell}$ is the corresponding continuous solution. Put differently, $\mathbf{U}_{\ell}$ is even a Galerkin approximation of $\widehat{\mathbf{U}}_{\ell}$, and the formulation above is the related Galerkin orthogonality. Consequently, quasi-optimality (2.17) of the Galerkin scheme proves

$$
\eta_{\ell}^{2}=\left\|\widehat{\mathbf{U}}_{\ell}-\mathbf{U}_{\ell}\right\|^{2} \lesssim \min _{\mathbf{V}_{\ell} \in \mathcal{X}_{\ell}}\left\|\widehat{\mathbf{U}}_{\ell}-\mathbf{V}_{\ell}\right\|^{2} \leq\left\|\left(1-I_{\ell}^{\Omega}\right) \widehat{U}_{\ell}\right\|_{H^{1}(\Omega)}^{2}+\left\|\left(1-\Pi_{\ell}^{\Gamma}\right) \widehat{\Phi}_{\ell}\right\|_{\mathfrak{V}^{j}}^{2}
$$

Note that $\left(1-I_{\ell}^{\Omega}\right) c=0$ for each constant function $c \in \mathbb{R}$. Therefore, a scaling argument yields

$$
\left\|\left(1-I_{\ell}^{\Omega}\right) \widehat{U}_{\ell}\right\|_{H^{1}(T)} \lesssim\left\|\nabla\left(1-I_{\ell}^{\Omega}\right) \widehat{U}_{\ell}\right\|_{L^{2}(T)}=\mu_{\ell}(T) .
$$

Finally, it is an approximation result from [9], Theorem 4.1, Lemma 4.3, that

$$
\left\|\left(1-\Pi_{\ell}^{\Gamma}\right) \widehat{\Phi}_{\ell}\right\|_{\mathfrak{V}} \lesssim\left\|h_{\ell}^{1 / 2}\left(1-\Pi_{\ell}^{\Gamma}\right) \widehat{\Phi}_{\ell}\right\|_{L^{2}(\Gamma)},
$$

where the constant depends only on $\Gamma$, see also [18], Lemma 2.1. The combination of the last three estimates thus yields $\eta_{\ell} \lesssim \mu_{\ell}$ and concludes the proof.

Theorem 3.9. There is a constant $C_{13}>0$ which depends only on $\Omega, \kappa\left(\mathcal{E}_{0}\right)$, and $\sigma\left(\mathcal{T}_{0}\right)$ such that

$$
C_{13}^{-1} \mu_{\ell} \leq\left\|\mathbf{u}-\mathbf{U}_{\ell}\right\|+\text { osc }_{\ell} .
$$

Under the saturation assumption (3.13), there is a constant $C_{14}>0$ such that

$$
C_{14}^{-1}\left\|\mathbf{u}-\mathbf{U}_{\ell}\right\| \leq \mu_{\ell}+\operatorname{osc}_{\ell} .
$$

Besides $\Omega$ and $\kappa\left(\mathcal{E}_{0}\right)$, the constant $C_{14}>0$ depends only on $0<C_{\mathrm{sat}}<1$. The same estimates hold for $\widetilde{\mu}_{\ell}$ replacing $\mu_{\ell}$.

Proof. The proof is an obvious consequence of Proposition 3.7 and the equivalence of the introduced error estimators stated in Lemma 3.8.

\section{Convergent adaptive COUpling}

\subsection{A priori convergence of adaptive algorithms}

Before we state the adaptive algorithm and prove convergence of which, we claim the a priori convergence of adaptive mesh-refining algorithms. By this, we mean that the sequences $\mathbf{U}_{\ell}^{\star}$ and $\mathbf{U}_{\ell}$ of discrete solutions always tend to certain limits $\mathbf{u}_{\infty}^{\star}$ and $\mathbf{u}_{\infty}$, independently of how the mesh is actually refined. Note carefully, however, that we do not claim that $\mathbf{u}$ coincides with one of the a priori limits $\mathbf{u}_{\infty}^{\star}$ or $\mathbf{u}_{\infty}$.

To deal with the data approximation, we need the following convergence result, which will be applied for the approximate data $U_{0, \ell}^{\prime}=\left(I_{\ell}^{\Gamma} u_{0}\right)^{\prime}=\Pi_{\ell}^{\Gamma}\left(u_{0}^{\prime}\right) \in H:=L^{2}(\Gamma)$. A proof can be found in $[4,12,26]$ or even in the early work [5], Lemma 6.1 . 
Lemma 4.1. Suppose that $H$ is a Hilbert space and $\left(X_{\ell}\right)_{\ell \in \mathbb{N}}$ is a sequence of closed subspaces of $H$ with $X_{\ell} \subseteq X_{\ell+1}$. Let $\mathbb{P}_{\ell}: H \rightarrow X_{\ell}$ denote the orthogonal projection onto $X_{\ell}$. Then, for any $x \in H$ and $x_{\ell}:=\mathbb{P}_{\ell} x$, the limit $x_{\infty}:=\lim _{\ell \rightarrow \infty} x_{\ell} \in H$ exists.

Proposition 4.2. Let $\mathcal{T}_{\ell}$ and $\mathcal{E}_{\ell}$ be a sequence of meshes with corresponding nested spaces

$$
\mathcal{X}_{\ell} \subseteq \mathcal{X}_{\ell+1}
$$

Let $\mathbf{U}_{\ell}^{\star} \in \mathcal{X}_{\ell}$ and $\mathbf{U}_{\ell} \in \mathcal{X}_{\ell}$ be the corresponding Galerkin solutions of (2.14) and (2.20), respectively. Then, there are limits $\mathbf{u}_{\infty}^{\star}, \mathbf{u}_{\infty} \in \mathcal{H}$ such that

$$
\lim _{\ell \rightarrow \infty}\left\|\mathbf{u}_{\infty}^{\star}-\mathbf{U}_{\ell}^{\star}\right\|=0=\lim _{\ell \rightarrow \infty}\left\|\mathbf{u}_{\infty}-\mathbf{U}_{\ell}\right\| .
$$

Proof of a priori convergence of $\mathbf{U}_{\ell}^{\star}$. We define the space $\mathcal{X}_{\infty}$ as the closure of $\bigcup_{\ell=0}^{\infty} \mathcal{X}_{\ell}$. Then, $\mathcal{X}_{\infty}$ is a closed subspace of $\mathcal{H}$ which thus admits a unique Galerkin solution $\mathbf{u}_{\infty}^{\star} \in \mathcal{X}_{\infty}$ of (2.14), see Appendix A. Arguing as in the proof of Lemma 3.8, we see that $\mathbf{U}_{\ell}^{\star} \in \mathcal{X}_{\ell}$ is also a Galerkin approximation of $\mathbf{u}_{\infty}^{\star}$ and that there holds the Céa lemma

$$
\left\|\mathbf{u}_{\infty}^{\star}-\mathbf{U}_{\ell}^{\star}\right\| \lesssim \min _{\mathbf{V}_{\ell} \in \mathcal{X}_{\ell}}\left\|\mathbf{u}_{\infty}^{\star}-\mathbf{V}_{\ell}\right\| .
$$

Let $\varepsilon>0$. By definition of $\mathcal{X}_{\infty}$, we find some index $\ell_{0} \in \mathbb{N}$ and some function $\mathbf{V}_{\ell_{0}} \in \mathcal{X}_{\ell_{0}}$ such that $\| \mathbf{u}_{\infty}^{\star}-$ $\mathbf{V}_{\ell_{0}} \| \leq \varepsilon$. From nestedness of $\mathcal{X}_{\ell}$, we infer

$$
\left\|\mathbf{u}_{\infty}^{\star}-\mathbf{U}_{\ell}^{\star}\right\| \lesssim\left\|\mathbf{u}_{\infty}^{\star}-\mathbf{V}_{\ell_{0}}\right\| \leq \varepsilon
$$

for all $\ell \geq \ell_{0}$. This proves convergence $\lim _{\ell \rightarrow \infty} \mathbf{U}_{\ell}^{\star}=\mathbf{u}_{\infty}^{\star}$.

Proof of a priori convergence of $\mathbf{U}_{\ell}$. In $1 \mathrm{D}$, the nodal interpolation operator $I_{\ell}^{\Gamma}: H^{1}(\Gamma) \rightarrow \mathcal{S}^{1}\left(\mathcal{E}_{\ell}\right)$ and the $L^{2}$ orthogonal projection $\Pi_{\ell}^{\Gamma}: L^{2}(\Gamma) \rightarrow \mathcal{P}^{0}\left(\mathcal{E}_{\ell}\right)$ are linked through the identity $\left(I_{\ell}^{\Gamma} v\right)^{\prime}=\Pi_{\ell}^{\Gamma}\left(v^{\prime}\right)$, cf. Lemma 3.3. From nestedness $\mathcal{P}^{0}\left(\mathcal{E}_{\ell}\right) \subseteq \mathcal{P}^{0}\left(\mathcal{E}_{\ell+1}\right)$, one may thus derive that the $L^{2}$-limit

$$
g=\lim _{\ell \rightarrow \infty} U_{0, \ell}^{\prime}=\lim _{\ell \rightarrow \infty} \Pi_{\ell}^{\Gamma}\left(u_{0}^{\prime}\right) \in L^{2}(\Gamma)
$$

exists, see Lemma 4.1. In particular, the sequence $\left(U_{0, \ell}^{\prime}\right)_{\ell \in \mathbb{N}}$ of derivatives is a Cauchy sequence in $L^{2}(\Gamma)$. Let $\ell \geq k$. With the help of Lemma 3.3, we see

$$
\begin{aligned}
\left\|U_{0, \ell}-U_{0, k}\right\|_{H^{1 / 2}(\Gamma)} & =\left\|\left(1-I_{k}^{\Gamma}\right) U_{0, \ell}\right\|_{H^{1 / 2}(\Gamma)} \lesssim\left\|h_{\ell}^{1 / 2}\left[\left(1-I_{k}^{\Gamma}\right) U_{0, \ell}\right]^{\prime}\right\|_{L^{2}(\Gamma)} \\
& =\left\|h_{\ell}^{1 / 2}\left(U_{0, \ell}^{\prime}-U_{0, k}^{\prime}\right)\right\|_{L^{2}(\Gamma)} \\
& \lesssim\left\|U_{0, \ell}^{\prime}-U_{0, k}^{\prime}\right\|_{L^{2}(\Gamma)} \stackrel{k, \ell \rightarrow \infty}{\longrightarrow} 0 .
\end{aligned}
$$

Consequently, the sequence $\left(U_{0, \ell}\right)_{\ell \in \mathbb{N}}$ is a Cauchy sequence in $H^{1 / 2}(\Gamma)$ and thus convergent to some $H^{1 / 2}$-limit

$$
u_{0, \infty}=\lim _{\ell \rightarrow \infty} U_{0, \ell} \in H^{1 / 2}(\Gamma) .
$$

We now consider an auxiliary problem, where we only replace $u_{0}$ in the variational formulation (2.3) and its Galerkin discretization $(2.14)$ by the obtained limit $u_{0, \infty}$. This provides a sequence $\mathbf{U}_{\infty, \ell}^{\star} \in \mathcal{X}_{\ell}$ of Galerkin solutions. The already proven a priori convergence of $\mathbf{U}_{\ell}^{\star}$ applies to this auxiliary problem as well. Consequently, the $\mathcal{H}$-limit

$$
\mathbf{u}_{\infty}=\lim _{\ell \rightarrow \infty} \mathbf{U}_{\infty, \ell}^{\star} \in \mathcal{H}
$$


exists. The triangle inequality proves

$$
\left\|\mathbf{u}_{\infty}-\mathbf{U}_{\ell}\right\| \leq\left\|\mathbf{u}_{\infty}-\mathbf{U}_{\infty, \ell}^{\star}\right\|\|+\| \mathbf{U}_{\infty, \ell}^{\star}-\mathbf{U}_{\ell} \| .
$$

The first summand is known to tend to zero by definition of $\mathbf{u}_{\infty}$. For the second summand, we apply Lemma 3.2 to see

$$
\left\|\mathbf{U}_{\infty, \ell}^{\star}-\mathbf{U}_{\ell}\right\| \lesssim\left\|u_{0, \infty}-U_{0, \ell}\right\|_{H^{1 / 2}(\Gamma)} \stackrel{\ell \rightarrow \infty}{\longrightarrow} 0
$$

by definition of $u_{0, \infty}$. This concludes the proof.

\subsection{Marking criterion and estimator reduction}

The marking is based on the Dörfler marking introduced in [16]. In view of Theorem 3.9, we consider the following refinement indicator

$$
\varrho_{\ell}(\tau)^{2}:= \begin{cases}\mu_{\ell}(T)^{2} & \text { for } \tau=T \in \mathcal{T}_{\ell}, \\ \mu_{\ell}(E)^{2}+\operatorname{osc}_{\ell}(E)^{2} & \text { for } \tau=E \in \mathcal{E}_{\ell},\end{cases}
$$

where the local data oscillations read

$$
\operatorname{osc}_{\ell}(E)^{2}=\operatorname{diam}(E)\left\|\left(u_{0}-U_{\ell, 0}\right)^{\prime}\right\|_{L^{2}(E)}^{2} \quad \text { for } E \in \mathcal{E}_{\ell} .
$$

By definition, there holds

$$
\varrho_{\ell}^{2}:=\mu_{\ell}^{2}+\operatorname{osc}_{\ell}^{2}=\sum_{\tau \in \mathcal{T}_{\ell} \cup \mathcal{E}_{\ell}} \varrho_{\ell}(\tau)^{2}
$$

For an arbitrary but fixed parameter $\theta \in(0,1)$, we then determine a set $\mathcal{M}_{\ell} \subseteq \mathcal{T}_{\ell} \cup \mathcal{E}_{\ell}$ of marked elements with

$$
\theta \varrho_{\ell}^{2} \leq \sum_{\tau \in \mathcal{M}_{\ell}} \varrho_{\ell}(\tau)^{2}
$$

Based on the Dörfler marking (4.5) and the mesh-refinement rule, we next prove the crucial estimator reduction which is, however, not stated for $\varrho_{\ell}$ but for $\widetilde{\varrho}_{\ell}=\left(\widetilde{\mu}_{\ell}^{2}+\operatorname{osc}_{\ell}^{2}\right)^{1 / 2}$.

Lemma 4.3. There are constants $\kappa \in(0,1)$ and $C_{15}>0$ such that

$$
\widetilde{\varrho}_{\ell+1}^{2} \leq \kappa \widetilde{\varrho}_{\ell}^{2}+C_{15}\left\|\widehat{\mathbf{U}}_{\ell+1}-\widehat{\mathbf{U}}_{\ell}\right\|^{2},
$$

where $\widetilde{\varrho}_{\ell}^{2}:=\widetilde{\mu}_{\ell}^{2}+\operatorname{osc}_{\ell}^{2}$. The contraction constant $\kappa \in(0,1)$ depends only on the adaptivity parameter $\theta \in(0,1)$, whereas the constant $C_{15}>0$ additionally depends on $\Omega$ and $\kappa\left(\mathcal{E}_{0}\right)$.

Proof. Recall the identity $U_{0, \ell}^{\prime}=\left(I_{\ell}^{\Gamma} u_{0}\right)^{\prime}=\Pi_{\ell}^{\Gamma}\left(u_{0}^{\prime}\right)$. First, for arbitrary $\delta>0$, the Young inequality proves

$$
\begin{aligned}
\widetilde{\varrho}_{\ell+1}^{2}= & \left\|\left(1-\Pi_{\ell+1}^{\Omega}\right) \nabla \widehat{U}_{\ell+1}\right\|_{L^{2}(\Omega)}^{2}+\left\|h_{\ell+1}^{1 / 2}\left(1-\Pi_{\ell+1}^{\Gamma}\right) \widehat{\Phi}_{\ell+1}\right\|_{L^{2}(\Gamma)}^{2} \\
& +\left\|h_{\ell+1}^{1 / 2}\left(1-\Pi_{\ell+1}^{\Gamma}\right) u_{0}^{\prime}\right\|_{L^{2}(\Gamma)}^{2} \\
\leq & (1+\delta)\left(\left\|\left(1-\Pi_{\ell+1}^{\Omega}\right) \nabla \widehat{U}_{\ell}\right\|_{L^{2}(\Omega)}^{2}+\left\|h_{\ell+1}^{1 / 2}\left(1-\Pi_{\ell+1}^{\Gamma}\right) \widehat{\Phi}_{\ell}\right\|_{L^{2}(\Gamma)}^{2}\right) \\
& +\left(1+\delta^{-1}\right)\left(\left\|\nabla \widehat{U}_{\ell+1}-\nabla \widehat{U}_{\ell}\right\|_{L^{2}(\Omega)}^{2}+\left\|h_{\ell+1}^{1 / 2}\left(\widehat{\Phi}_{\ell+1}-\widehat{\Phi}_{\ell}\right)\right\|_{L^{2}(\Gamma)}^{2}\right) \\
& +\left\|h_{\ell+1}^{1 / 2}\left(1-\Pi_{\ell+1}^{\Gamma}\right) u_{0}^{\prime}\right\|_{L^{2}(\Gamma)}^{2},
\end{aligned}
$$


where we have additionally used that all of the involved $L^{2}$-orthogonal projections are even elementwise best approximation operators. Second, for $T \in \mathcal{T}_{\ell} \cap \mathcal{M}_{\ell}$ holds

$$
\left\|\left(1-\Pi_{\ell+1}^{\Omega}\right) \nabla \widehat{U}_{\ell}\right\|_{L^{2}(T)}^{2}=0,
$$

whereas for $T \in \mathcal{T}_{\ell} \backslash \mathcal{M}_{\ell}$ holds

$$
\left\|\left(1-\Pi_{\ell+1}^{\Omega}\right) \nabla \widehat{U}_{\ell}\right\|_{L^{2}(T)}^{2} \leq \widetilde{\mu}_{\ell}(T)^{2} .
$$

Note that $\leq$ in the last estimate stems from the fact that an element $T \in \mathcal{T}_{\ell} \backslash \mathcal{M}_{\ell}$ may be refined to avoid hanging nodes, $c f$. Section 3.1. For $E \in \mathcal{E}_{\ell} \cap \mathcal{M}_{\ell}$ holds

$$
\begin{aligned}
\left\|h_{\ell+1}^{1 / 2}\left(1-\Pi_{\ell+1}^{\Gamma}\right) \widehat{\Phi}_{\ell}\right\|_{L^{2}(E)}^{2}+\left\|h_{\ell+1}^{1 / 2}\left(1-\Pi_{\ell+1}^{\Gamma}\right) u_{0}^{\prime}\right\|_{L^{2}(E)}^{2} & =\frac{1}{2}\left\|h_{\ell}^{1 / 2}\left(1-\Pi_{\ell+1}^{\Gamma}\right) u_{0}^{\prime}\right\|_{L^{2}(E)}^{2} \\
& \leq \frac{1}{2}\left(\widetilde{\mu}_{\ell}(E)^{2}+\operatorname{osc}_{\ell}(E)^{2}\right),
\end{aligned}
$$

whereas for $E \in \mathcal{E}_{\ell} \backslash \mathcal{M}_{\ell}$ holds

$$
\left\|h_{\ell+1}^{1 / 2}\left(1-\Pi_{\ell+1}^{\Gamma}\right) \widehat{\Phi}_{\ell}\right\|_{L^{2}(E)}^{2}+\left\|h_{\ell+1}^{1 / 2}\left(1-\Pi_{\ell+1}^{\Gamma}\right) u_{0}^{\prime}\right\|_{L^{2}(E)}^{2} \leq \widetilde{\mu}_{\ell}(E)^{2}+\operatorname{osc}_{\ell}(E)^{2} .
$$

Third, the local estimates are used to obtain

$$
\begin{aligned}
\|(1- & \left.\Pi_{\ell+1}^{\Omega}\right) \nabla \widehat{U}_{\ell}\left\|_{L^{2}(\Omega)}^{2}+\right\| h_{\ell+1}^{1 / 2}\left(1-\Pi_{\ell+1}^{\Gamma}\right) \widehat{\Phi}_{\ell}\left\|_{L^{2}(\Gamma)}^{2}+\right\| h_{\ell+1}^{1 / 2}\left(1-\Pi_{\ell+1}^{\Gamma}\right) u_{0}^{\prime} \|_{L^{2}(\Gamma)}^{2} \\
& \leq \sum_{T \in \mathcal{T}_{\ell} \backslash \mathcal{M}_{\ell}} \widetilde{\mu}_{\ell}(T)^{2}+\frac{1}{2} \sum_{E \in \mathcal{E}_{\ell} \cap \mathcal{M}_{\ell}}\left(\widetilde{\mu}_{\ell}(E)^{2}+\operatorname{osc}_{\ell}(E)^{2}\right)+\sum_{E \in \mathcal{E}_{\ell} \backslash \mathcal{M}_{\ell}}\left(\widetilde{\mu}_{\ell}(E)^{2}+\operatorname{osc}_{\ell}(E)^{2}\right) \\
& \leq \sum_{T \in \mathcal{T}_{\ell}} \widetilde{\mu}_{\ell}(T)^{2}+\sum_{E \in \mathcal{E}_{\ell}}\left(\widetilde{\mu}_{\ell}(E)^{2}+\operatorname{osc}_{\ell}(E)^{2}\right)-\frac{1}{2}\left(\sum_{T \in \mathcal{T}_{\ell} \cap \mathcal{M}_{\ell}} \widetilde{\mu}_{\ell}(T)^{2}+\sum_{E \in \mathcal{E}_{\ell} \cap \mathcal{M}_{\ell}}\left(\widetilde{\mu}_{\ell}(E)^{2}+\operatorname{osc}_{\ell}(E)^{2}\right)\right) \\
& =\widetilde{\varrho}_{\ell}^{2}-\frac{1}{2}\left(\sum_{T \in \mathcal{T}_{\ell} \cap \mathcal{M}_{\ell}} \widetilde{\mu}_{\ell}(T)^{2}+\sum_{E \in \mathcal{E}_{\ell} \cap \mathcal{M}_{\ell}}\left(\widetilde{\mu}_{\ell}(E)^{2}+\operatorname{osc}_{\ell}(E)^{2}\right)\right) .
\end{aligned}
$$

Fourth, we employ the marking strategy (4.5) and the local equivalence (3.23) to see

$$
\theta \widetilde{\varrho}_{\ell}^{2} \leq \theta \varrho_{\ell}^{2} \leq \sum_{\tau \in \mathcal{M}_{\ell}} \varrho_{\ell}(\tau)^{2} \leq C_{10} \sum_{T \in \mathcal{T}_{\ell} \cap \mathcal{M}_{\ell}} \widetilde{\mu}_{\ell}(T)^{2}+\sum_{E \in \mathcal{E}_{\ell} \cap \mathcal{M}_{\ell}}\left(\widetilde{\mu}_{\ell}(E)^{2}+\operatorname{osc}_{\ell}(E)^{2}\right) .
$$

From $C_{10} \geq 1$, we thus infer

$$
\widetilde{\theta} \widetilde{\varrho}_{\ell}^{2} \leq \sum_{T \in \mathcal{T}_{\ell} \cap \mathcal{M}_{\ell}} \widetilde{\mu}_{\ell}(T)^{2}+\sum_{E \in \mathcal{E}_{\ell} \cap \mathcal{M}_{\ell}}\left(\widetilde{\mu}_{\ell}(E)^{2}+\operatorname{osc}_{\ell}(E)^{2}\right)
$$

with $\widetilde{\theta}=\theta / C_{10} \in(0,1)$. We now combine all aforegoing estimates to see

$$
\widetilde{\varrho}_{\ell+1}^{2} \leq(1+\delta)(1-\tilde{\theta} / 2) \widetilde{\varrho}_{\ell}^{2}+\left(1+\delta^{-1}\right)\left(\left\|\widehat{U}_{\ell+1}-\widehat{U}_{\ell}\right\|_{H^{1}(\Omega)}^{2}+\left\|h_{\ell+1}^{1 / 2}\left(\widehat{\Phi}_{\ell+1}-\widehat{\Phi}_{\ell}\right)\right\|_{L^{2}(\Gamma)}^{2}\right) .
$$

Since $(1-\tilde{\theta} / 2)<1$, we may choose $\delta>0$ with $\kappa:=(1+\delta)(1-\tilde{\theta} / 2)<1$. Moreover, the local inverse estimate from [22], Theorem 3.6, proves

$$
\left\|h_{\ell+1}^{1 / 2}\left(\widehat{\Phi}_{\ell+1}-\widehat{\Phi}_{\ell}\right)\right\|_{L^{2}(\Gamma)} \lesssim\left\|\widehat{\Phi}_{\ell+1}-\widehat{\Phi}_{\ell}\right\|_{\mathfrak{V}},
$$

where the constant depends only on $\Gamma$ and an upper bound of $\kappa\left(\mathcal{E}_{\ell}\right)$. Plugging this into the last estimate, we finally end up with (4.6). 


\subsection{Convergent adaptive algorithm}

We now consider the following adaptive algorithm. We stress that an adaptive algorithm does neither know the a priori limit $\mathbf{u}_{\infty}=\left(u_{\infty}, \phi_{\infty}\right)$ nor $\widehat{\mathbf{u}}_{\infty}=\left(\widehat{u}_{\infty}, \widehat{\phi}_{\infty}\right)$.

Algorithm 4.4. InPUT: initial meshes $\left(\mathcal{T}_{0}, \mathcal{E}_{0}\right)$ for $\ell:=0$, adaptivity parameter $\theta \in(0,1)$.

(i) Generate uniformly refined meshes $\widehat{\mathcal{T}}_{\ell}, \widehat{\mathcal{E}}_{\ell}$;

(ii) compute discrete solution $\widehat{\mathbf{U}}_{\ell} \in \widehat{\mathcal{X}}_{\ell}$;

(iii) compute refinement indicators $\varrho_{\ell}(\tau)$ for all $\tau \in \mathcal{T}_{\ell} \cup \mathcal{E}_{\ell}$;

(iv) determine set $\mathcal{M}_{\ell} \subseteq \mathcal{T}_{\ell} \cup \mathcal{E}_{\ell}$ which satisfies Dörfler marking (4.5);

(v) Mark triangles $T \in \mathcal{T}_{\ell} \cap \mathcal{M}_{\ell}$ and boundary elements $E \in \mathcal{E}_{\ell} \cap \mathcal{M}_{\ell}$ for refinement.

(vi) generate new meshes $\left(\mathcal{T}_{\ell+1}, \mathcal{E}_{\ell+1}\right)$, increase counter $\ell \mapsto \ell+1$, and goto (i).

OutpuT: sequence of error estimators $\left(\varrho_{\ell}\right)_{\ell \in \mathbb{N}}$ and discrete solutions $\left(\widehat{\mathbf{U}}_{\ell}\right)_{\ell \in \mathbb{N}}$.

Theorem 4.5. Algorithm 4.4 enforces

$$
\lim _{\ell \rightarrow \infty} \varrho_{\ell}=0
$$

Under the saturation assumption (3.13), this implies convergence

$$
\lim _{\ell \rightarrow \infty}\left\|(u, \phi)-\left(\widehat{U}_{\ell}, \widehat{\Phi}_{\ell}\right)\right\|=0=\lim _{\ell \rightarrow \infty}\left\|(u, \phi)-\left(U_{\ell}, \Phi_{\ell}\right)\right\| .
$$

Proof. The idea of the proof goes back to [4]. We combine the estimator reduction (4.6) with the a priori convergence (4.1) and the estimator equivalence (3.24): first, we note that Lemma 4.3 provides an estimate of the type

$$
\widetilde{\varrho}_{\ell+1}^{2} \leq \kappa \widetilde{\varrho}_{\ell}^{2}+\alpha_{\ell}
$$

with some constant $\kappa \in(0,1)$. According to Proposition 4.2 applied for $\widehat{\mathcal{X}}_{\ell}$, the nonnegative sequence $\alpha_{\ell} \simeq$ $\left\|\widehat{\mathbf{U}}_{\ell+1}-\widehat{\mathbf{U}}_{\ell}\right\|^{2}$ tends to zero. It is a consequence of elementary calculus that this implies estimator convergence

$$
\lim _{\ell \rightarrow \infty} \widetilde{\varrho}_{\ell}=0
$$

Now, the equivalence of $\widetilde{\varrho}_{\ell} \simeq \varrho_{\ell}$ - as a consequence of Lemma 3.8 - concludes (4.7).

Under the saturation assumption (3.13), Proposition 3.7 states

$$
\left\|\mathbf{u}-\mathbf{U}_{\ell}\right\| \lesssim \mu_{\ell}+\operatorname{osc}_{\ell} \lesssim \varrho_{\ell} \stackrel{\ell \rightarrow \infty}{\longrightarrow} 0
$$

and thus convergence of $\mathbf{U}_{\ell}$ to $\mathbf{u}$. In this case, the quasi-optimality $\left\|\mathbf{u}-\widehat{\mathbf{U}}_{\ell}\right\| \lesssim\left\|\mathbf{u}-\mathbf{U}_{\ell}\right\|$ also predicts convergence of $\widehat{\mathbf{U}}_{\ell}$.

Remark 4.6. It is a consequence of Theorem 4.5 that one obtains additional information on the a priori limits from the proof of Proposition 4.2. For instance, $\lim _{\ell} \operatorname{osc}_{\ell}=0$ predicts $u_{0, \infty}=u_{0}$, whence $\mathbf{u}_{\infty}^{\star}=\mathbf{u}_{\infty}$ and $\widehat{\mathbf{u}}_{\infty}^{\star}=\widehat{\mathbf{u}}_{\infty}$ if the latter denote the a priori limits of $\widehat{\mathbf{U}}_{\ell}^{\star}$ and $\widehat{\mathbf{U}}_{\ell}$. Moreover, $\lim _{\ell} \mu_{\ell}=0$ implies $\lim _{\ell} \eta_{\ell}=0$ and thus even $\mathbf{u}_{\infty}=\widehat{\mathbf{u}}_{\infty}$. 


\section{NUMERICAL EXPERIMENTS}

In this section, we present three numerical examples from [13] to demonstrate the advantages of the proposed adaptive FEM-BEM coupling and its superiority over uniform mesh-refinement. In all experiments, we prescribe the exact solution $\left(u^{\mathrm{int}}, u^{\mathrm{ext}}\right)$ of the transmission problem $(2.1)$, and the data $\left(u_{0}, \phi_{0}, f\right)$ are computed thereof. To simplify the implementation, we only consider the induced boundary partition $\mathcal{E}_{\ell}:=\left.\mathcal{T}_{\ell}\right|_{\Gamma}$.

Note that the contribution $\left\|\phi-\Phi_{\ell}\right\|_{\mathfrak{V}}$ to the error $\left\|\mathbf{u}-\mathbf{U}_{\ell}\right\|$ can hardly be computed analytically. However, according to Proposition 3.1 and and the quasi optimality (2.17), there holds

$$
\left\|\mathbf{u}-\mathbf{U}_{\ell}\right\| \lesssim\left\|\mathbf{u}-\mathbf{U}_{\ell}^{\star}\right\|+\operatorname{osc}_{\ell} \lesssim\left\|u-U_{\ell}\right\|_{H^{1}(\Omega)}+\min _{\Psi_{\ell} \in \mathcal{P}^{0}\left(\mathcal{E}_{\ell}\right)}\left\|\phi-\Psi_{\ell}\right\|_{\mathfrak{V}}+\operatorname{osc}_{\ell}
$$

with $\mathbf{u}=(u, \phi)$ and $\mathbf{U}_{\ell}=\left(U_{\ell}, \Phi_{\ell}\right)$. In all experiments, the exterior normal derivative has additional regularity $\phi \in L^{2}(\Gamma)$. We may therefore proceed as in the proof of Lemma 3.8 to obtain

$$
\min _{\Psi_{\ell} \in \mathcal{P}^{0}\left(\mathcal{E}_{\ell}\right)}\left\|\phi-\Psi_{\ell}\right\|_{\mathfrak{V}} \leq\left\|\left(1-\Pi_{\ell}^{\Gamma}\right) \phi\right\|_{\mathfrak{V}} \lesssim\left\|h_{\ell}^{1 / 2}\left(1-\Pi_{\ell}^{\Gamma}\right) \phi\right\|_{L^{2}(\Gamma)} \leq\left\|h_{\ell}^{1 / 2}\left(\phi-\Phi_{\ell}\right)\right\|_{L^{2}(\Gamma)}
$$

with $\Pi_{\ell}^{\Gamma}: L^{2}(\Gamma) \rightarrow \mathcal{P}^{0}\left(\mathcal{E}_{\ell}\right)$ being the $L^{2}$-orthogonal projection. Altogether, we see that

$$
\begin{aligned}
\left\|\mathbf{u}-\mathbf{U}_{\ell}\right\| & \lesssim\left\|u-U_{\ell}\right\|_{H^{1}(\Omega)}+\left\|h_{\ell}^{1 / 2}\left(\phi-\Phi_{\ell}\right)\right\|_{L^{2}(\Gamma)}+\operatorname{osc}_{\ell} \\
& =: \operatorname{err}_{\ell}(u)+\operatorname{err}_{\ell}(\phi)+\operatorname{osc}_{\ell}
\end{aligned}
$$

provides an upper bound for the energy error. For a known exact solution $\mathbf{u}=(u, \phi)$, the latter bound is computable at least by means of numerical quadrature and thus allows to monitor the decay of the error independently of the saturation assumption. In the same spirit, the error estimator $\mu_{\ell}$ is split into

$$
\mu_{\ell}^{2}=\sum_{T \in \mathcal{T}_{\ell}} \mu_{\ell}(T)^{2}+\sum_{E \in \mathcal{E}_{\ell}} \mu_{\ell}(E)^{2}=: \mu_{\ell}(u)^{2}+\mu_{\ell}(\phi)^{2} .
$$

Recall that Theorem 3.9 predicts

$$
\mu_{\ell}(u)+\mu_{\ell}(\phi)+\operatorname{osc}_{\ell} \lesssim\left\|\mathbf{u}-\mathbf{U}_{\ell}\right\|+\operatorname{osc}_{\ell} \lesssim \mu_{\ell}(u)+\mu_{\ell}(\phi)+\operatorname{osc}_{\ell},
$$

where the upper bound holds under the saturation assumption (3.13).

In the following, we plot the five quantities $\operatorname{err}_{\ell}(u), \operatorname{err}_{\ell}(\phi), \mu_{\ell}(u), \mu_{\ell}(\phi)$, and $\operatorname{osc}_{\ell}$ from (5.1)-(5.2) over the number $N=\# \mathcal{T}_{\ell}$ of triangles, where both axes are scaled logarithmically. We consider uniform mesh-refinement $\mathcal{T}_{\ell}=\mathcal{T}_{\ell}^{\text {(unif) }}$ with $\mathcal{T}_{\ell}^{\text {(unif) }}:=\widehat{\mathcal{T}}_{\ell-1}, c f$. Section 3.1, as well as adaptive mesh-refinement, where the sequence of meshes $\mathcal{T}_{\ell}=\mathcal{T}_{\ell}^{(\text {adap })}$ is generated by Algorithm 4.4 with $\theta=0.25$. Note that a decay with slope $-\alpha$ indicates some dependence $\mathcal{O}\left(N^{-\alpha}\right)$. For uniform meshes with mesh-size $h$, this corresponds to $\mathcal{O}\left(h^{2 \alpha}\right)$. We stress that, by theory, an overall slope of $\alpha=1 / 2$ is thus optimal with P1-finite elements.

For the adaptive mesh-refinement of Algorithm 4.4, recall that all integral operators have to be computed with respect to the fine mesh $\widehat{\mathcal{T}}_{\ell}$. Therefore, one usually takes the improved approximation $\widehat{U}_{0, \ell} \in \mathcal{S}^{1}\left(\left.\widehat{\mathcal{T}}_{\ell}\right|_{\Gamma}\right)$ instead of $U_{0, \ell} \in \mathcal{S}^{1}\left(\left.\mathcal{T}_{\ell}\right|_{\Gamma}\right)$. Consequently, we then consider

$$
\widehat{\mathrm{OSc}}_{\ell}=\left\|h_{\ell}^{1 / 2}\left(u_{0}-\widehat{U}_{0, \ell}\right)^{\prime}\right\|_{L^{2}(\Gamma)}
$$

instead of $\operatorname{osc}_{\ell}$. We stress that all results of this paper hold with osc $\ell$ replaced by $\widehat{o s c}_{\ell}$ as well. Moreover, although $\mathbf{U}_{\ell}$ is not needed by Algorithm 4.4, we nevertheless plot err $\ell$ to give a fair comparison of uniform and adaptive mesh-refinement.

Besides the experimental convergence rates, we plot $\operatorname{err}_{\ell}(u), \operatorname{err}_{\ell}(\phi), \mu_{\ell}(u), \mu_{\ell}(\phi)$, and $\operatorname{osc}_{\ell}\left(\operatorname{resp} . \widehat{o s c}_{\ell}\right) \operatorname{over}$ the computational time $t_{\ell}$. 

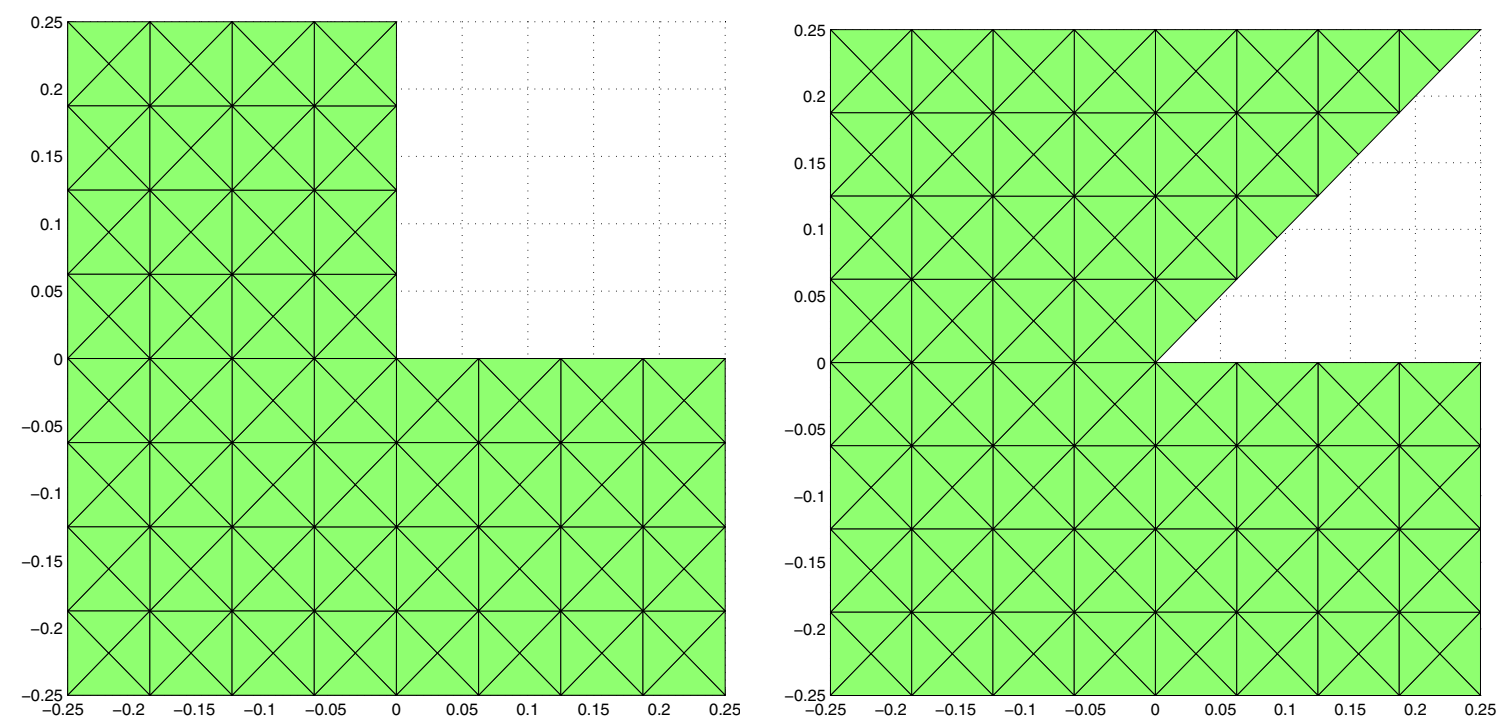

Figure 1. L-shaped (left) and Z-shaped domain (right) and initial triangulations $\mathcal{T}_{0}$ for the numerical experiments.

- For uniform mesh-refinement, $t_{\ell}=t_{\ell}^{\text {(unif) }}$ is the time needed for $\ell$ uniform refinements of the initial mesh $\mathcal{T}_{0}$ to obtain $\mathcal{T}_{\ell}$, plus the time for building and solving the Galerkin system with respect to $\mathcal{X}_{\ell}$.

For adaptive mesh-refinement, the mesh $\mathcal{T}_{\ell}$ depends on the entire history of preceding meshes (and solutions). Therefore, the computational time has to be defined differently, where $t_{-1}^{(\text {adap })}:=0$.

- For adaptive mesh-refinement, $t_{\ell}=t_{\ell}^{\text {(adap) }}$ is the sum of the time $t_{\ell-1}^{\text {(adap) }}$ elapsed in prior steps of the adaptive algorithm, plus the time for generating the fine mesh $\widehat{\mathcal{T}}_{\ell}$, building and solving the Galerkin system with respect to $\widehat{\mathcal{X}}_{\ell}$, computing the local contributions of the data oscillations $\widehat{\mathrm{osc}}_{\ell}$ and the error estimator $\mu_{\ell}$, element marking, and local refinement of $\mathcal{T}_{\ell}$ to generate $\mathcal{T}_{\ell+1}$.

Although this definition seems to favour uniform mesh-refinement, we think that it provides a fair comparison between uniform and adaptive mesh-refinement.

All experiments are conducted by use of MATLAB (Release 2009b) running on a common 64 Bit Linux system with 32 GB of RAM. Throughout, the occuring linear systems are solved by use of the MATLAB backslash operator. For the computation of the boundary integral operators, we use the MATLAB BEM library HILBERT, cf. [3]; see http://www.asc.tuwien.ac.at/abem/hilbert/

\subsection{Linear problem on L-shaped domain}

We consider the L-shaped domain visualized in Figure 1. With $A: L^{2}(\Omega)^{2} \rightarrow L^{2}(\Omega)^{2}$ being the identity, we prescribe the exact solution of (2.1) as

$$
\begin{array}{ll}
u^{\mathrm{int}}(x, y)=r^{2 / 3} \sin \left(\frac{2}{3} \varphi\right) & \text { in } \Omega^{\mathrm{int}}, \\
u^{\mathrm{ext}}(x, y)=\log \left(\left(x+\frac{1}{8}\right)^{2}+\left(y+\frac{1}{8}\right)^{2}\right)^{1 / 2} \text { in } \Omega^{\mathrm{ext}},
\end{array}
$$



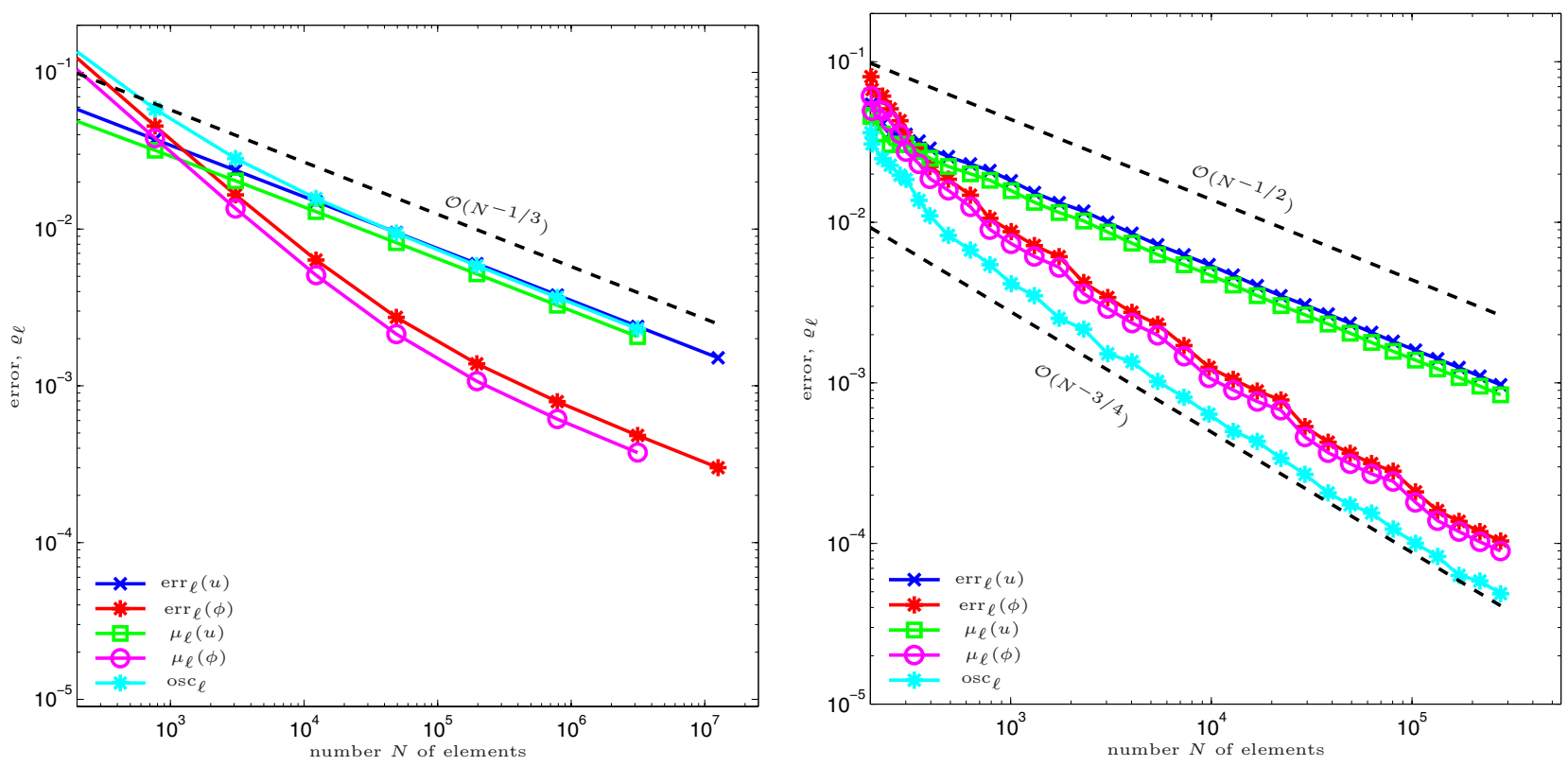

Figure 2. Estimators $\operatorname{err}_{\ell}(u), \operatorname{err}_{\ell}(\phi), \mu_{\ell}(u)$ and $\mu_{\ell}(\phi)$ from (5.1)-(5.2) as well as data oscillations osc $_{\ell}$ in linear experiment 5.1, plotted over the number $N=\# \mathcal{T}_{\ell}$ of triangles for uniform (left) and adaptive mesh-refinement (right).

where $(r, \varphi)$ are the polar coordinates of $(x, y) \in \mathbb{R}^{2}$ with respect to $(0,0)$. Clearly, the identity satisfies the assumptions of our model problem, and the FEM-BEM coupling $(2.3)$ is linear. Recall that $(u, \phi)$ denotes the exact solution of $(2.3)$ and note that $u=u^{\text {int }} \in H^{1+2 / 3-\varepsilon}(\Omega)$ for all $\varepsilon>0$ has a generic singularity at the reentrant corner, whereas $\phi=\nabla u^{\mathrm{ext}} \cdot n$ is piecewise smooth.

In Figure 2, we plot the convergence of the error quantities from (5.1)-(5.2). Since the interior solution has a generic singularity at the reentrant corner, uniform mesh-refinement leads to a suboptimal order of convergence $\alpha=1 / 3$, i.e. we observe $\mathcal{O}\left(h^{2 / 3}\right)$. For $\operatorname{err}_{\ell}(u)$ and $\mu_{\ell}(u)$, this asymptotics is observed already on coarse meshes. For $\operatorname{err}_{\ell}(\phi)$ and $\mu_{\ell}(\phi)$, a preasymptotic phase occurs. For adaptive mesh-refinement, we observe the optimal order of convergence $\alpha=1 / 2$ for $\operatorname{err}_{\ell}(u)$ and $\mu_{\ell}(u)$. Moreover, the terms $\operatorname{err}_{\ell}(\phi)$ and $\mu_{\ell}(\phi)$ even converge with order $\alpha=3 / 4$ which is optimal for the approximation of a smooth function by piecewise constants with respect to the $H^{-1 / 2}(\Gamma)$-norm.

Figure 3 provides comparisons between uniform and adaptive mesh-refinement. We plot

$$
\operatorname{err}_{\ell}:=\left(\operatorname{err}_{\ell}(u)^{2}+\operatorname{err}_{\ell}(\phi)^{2}+\operatorname{osc}_{\ell}^{2}\right)^{1 / 2} \text { and } \varrho_{\ell}=\left(\mu_{\ell}(u)^{2}+\mu_{\ell}(\phi)^{2}+\operatorname{osc}_{\ell}^{2}\right)^{1 / 2}
$$

over the number $N=\# \mathcal{T}_{\ell}$ of elements as well as over the computational time. Both plots underline that the proposed adaptive algorithm is much superior to uniform mesh-refinement.

\subsection{Nonlinear problem on L-shaped domain}

We consider the L-shaped domain visualized in Figure 1. We define

$$
\rho(t)=2+\frac{1}{1+t} \text { for } t>0
$$

and note that the derivative satisfies $-1 \leq \rho^{\prime}(t)<0$. The nonlinear operator $A$ is then defined by

$$
A(x)=\rho(|x|) x \quad \text { for } x \in \mathbb{R}^{2} .
$$



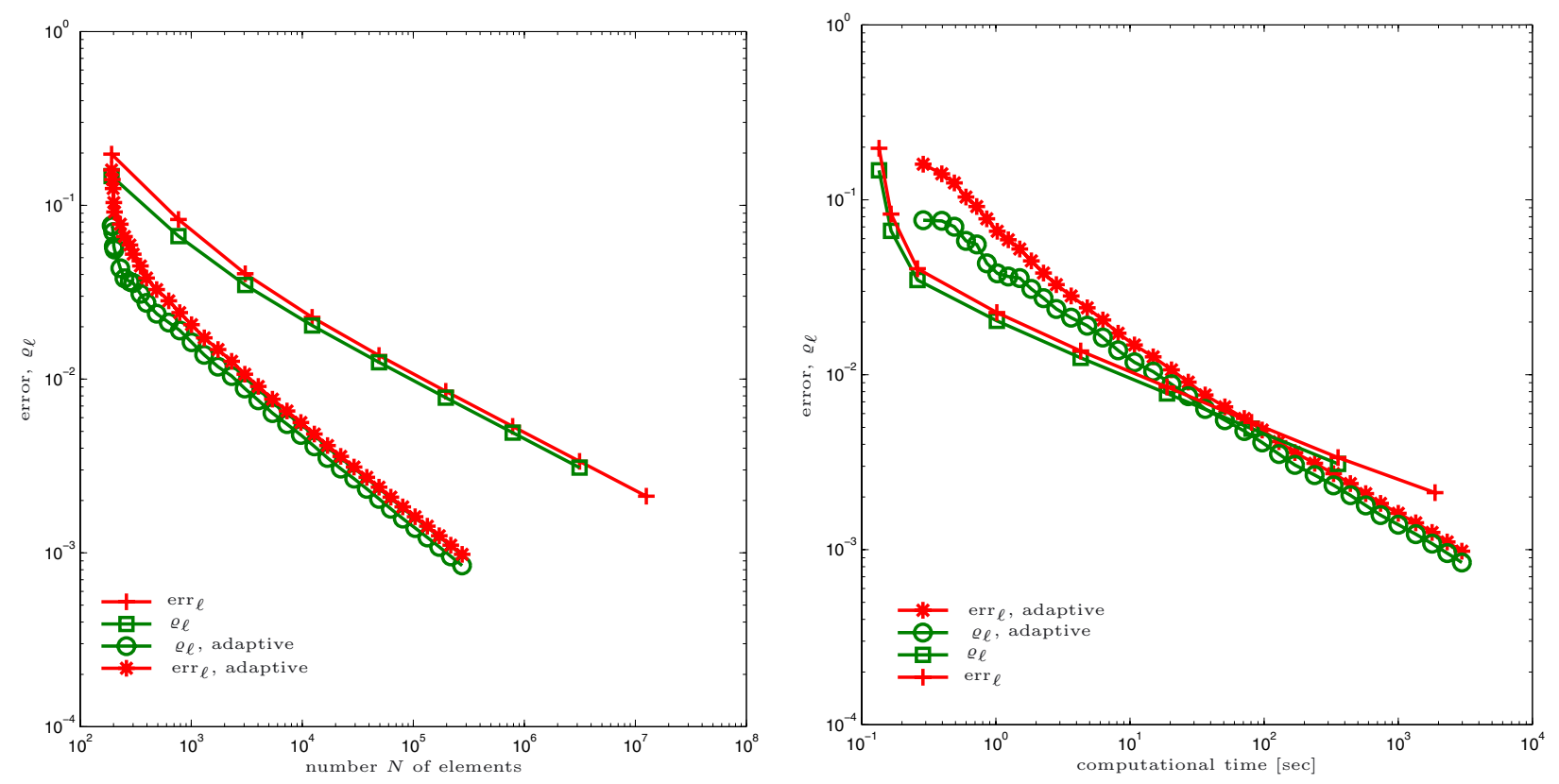

FiguRE 3. Comparison of uniform and adaptive mesh-refining in linear experiment 5.1, where the error bound $\operatorname{err}_{\ell}$ and the estimator $\varrho_{\ell}$ from (5.5) are plotted over the number $N=\# \mathcal{T}_{\ell}$ of triangles (left) and over the computational time (right).

We stress that $A$ is strongly monotone and Lipschitz continuous on $L^{2}(\Omega)$. We prescribe the same solution (5.4) as for the linear experiment from the previous section. The volume force $f$ then reads

$$
f(x, y):=-\frac{4}{27} r^{-5 / 3} \frac{\sin \left(\frac{2}{3} \varphi\right)}{\left(1+\frac{2}{3} r^{-1 / 3}\right)^{2}},
$$

where $(r, \varphi)$ are the polar coordinates of $(x, y) \in \mathbb{R}^{2}$ with respect to $(0,0)$.

The nonlinear system equivalent to $(2.20)$ is solved by an undamped Newton method. In our implementation, we computationally check that we are in the (quadratically convergent) asymptotic regime and stop the iteration if the Euclidean norm of the Newton residual increases. - Note that theory predicts the decay of the residual norms within the asymptotic regime. - For the initial mesh $\mathcal{T}_{0}$, the initial guess for the Newton scheme is the constant function $\mathbf{U}_{0}^{(0)} \equiv 1$. For the generated meshes $\mathcal{T}_{\ell}$, the initial guess is the preceding Galerkin approximation, i.e. $\mathbf{U}_{\ell}^{(0)}:=\mathbf{U}_{\ell-1}$, which is prolongated to the discrete space $\mathcal{X}_{\ell}$.

Figures 4 and 5 provide the experimental convergence results. The observations are the same as for the linear experiment in Section 5.1. Figure 6 shows some adaptively generated meshes. We observe a strong meshrefinement towards the reentrant corner, where $u^{\text {int }}$ is singular.

\subsection{Nonlinear problem on Z-shaped domain}

In the final example, $\Omega$ is the Z-shaped domain, shown in Figure 1. The exact solution reads

$$
\begin{array}{ll}
u^{\mathrm{int}}(x, y)=r^{4 / 7} \sin \left(\frac{4}{7} \varphi\right) & \text { in } \Omega^{\mathrm{int}}, \\
u^{\mathrm{ext}}(x, y)=\log \left(\left(x+\frac{1}{8}\right)^{2}+\left(y+\frac{1}{8}\right)^{2}\right)^{1 / 2} \text { in } \Omega^{\mathrm{ext}},
\end{array}
$$



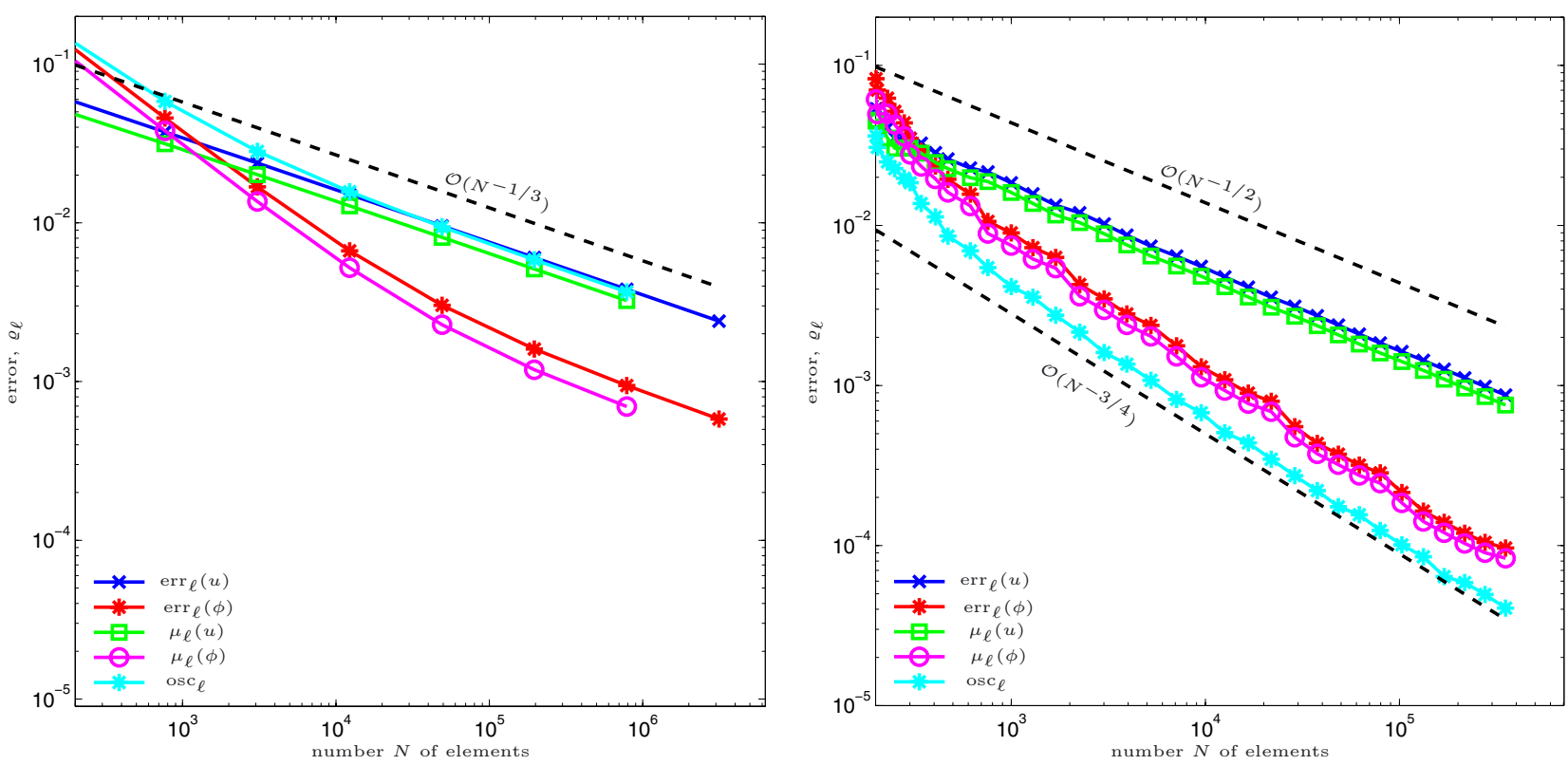

FIGURE 4. Estimators $\operatorname{err}_{\ell}(u), \operatorname{err}_{\ell}(\phi), \mu_{\ell}(u)$ and $\mu_{\ell}(\phi)$ from (5.1)-(5.2) as well as data oscillations osc $_{\ell}$ in nonlinear experiment 5.2 on the L-shaped domain, plotted over the number $N=\# \mathcal{T}_{\ell}$ of triangles for uniform (left) and adaptive mesh-refinement (right).
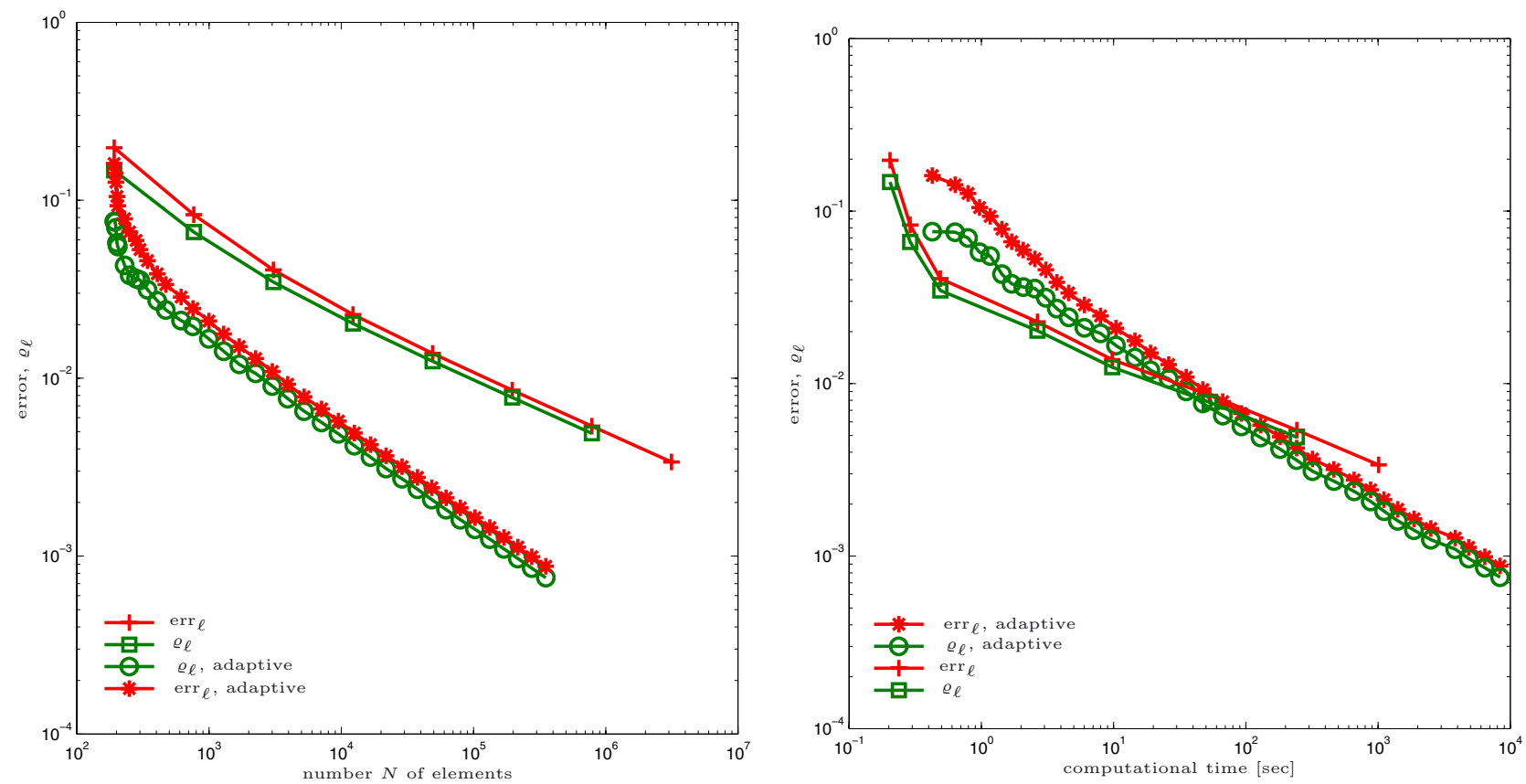

FigURE 5. Comparison of uniform and adaptive mesh-refining in nonlinear experiment 5.2 on the L-shaped domain, where the error bound $\operatorname{err}_{\ell}$ and the estimator $\varrho_{\ell}$ from (5.5) are plotted over the number $N=\# \mathcal{T}_{\ell}$ of triangles (left) and over the computational time (right). 

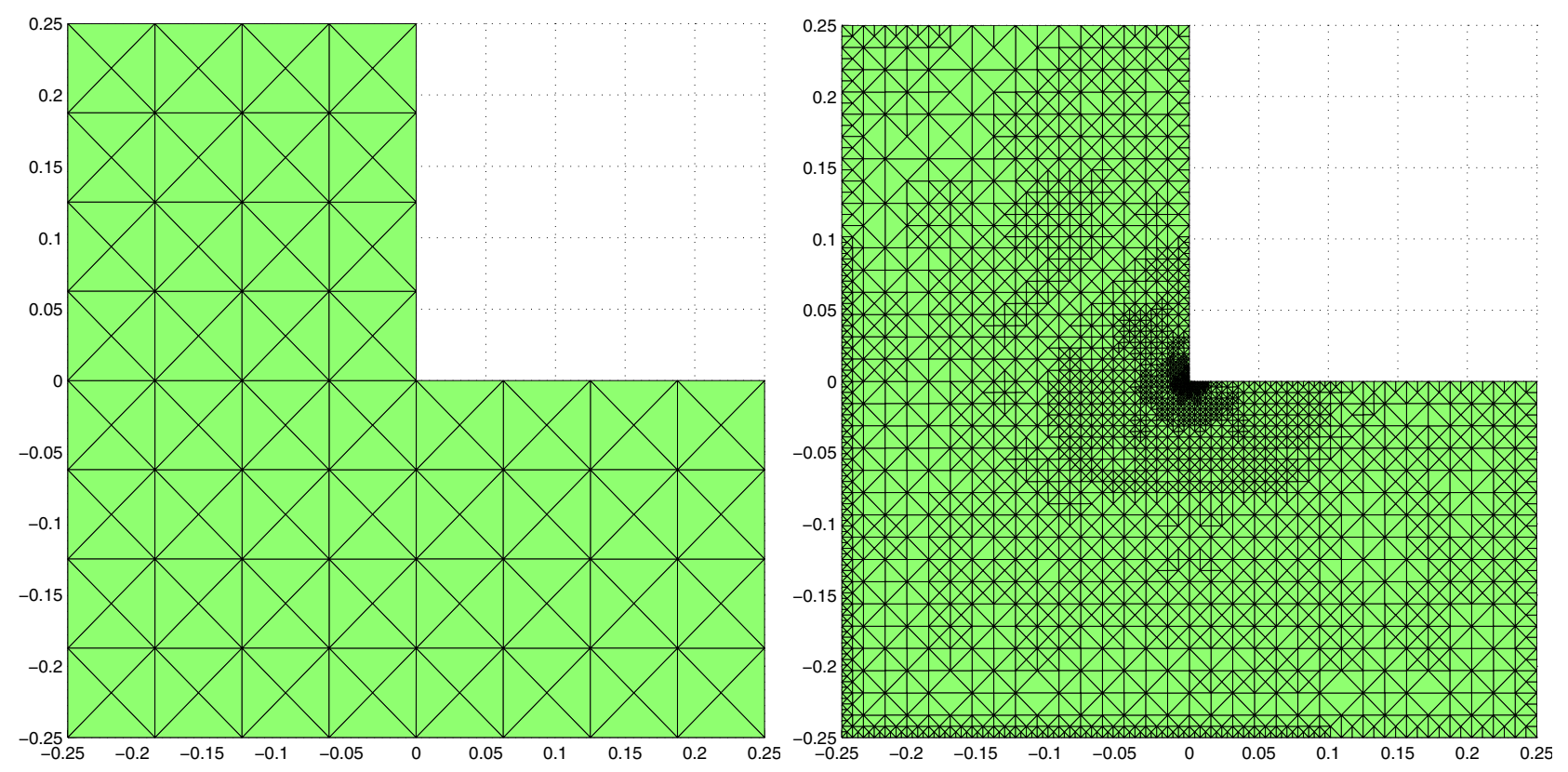

Figure 6 . Adaptively generated meshes $\mathcal{T}_{\ell}$ in nonlinear experiment 5.2 on the L-shaped domain.

with $(r, \varphi)$ being the polar coordinates of $(x, y) \in \mathbb{R}^{2}$. Again, $u^{\text {int }} \in H^{1+4 / 7-\varepsilon}(\Omega)$ for all $\varepsilon>0$ has a generic singularity at the reentrant corner. With the nonlinear operator $A$ from Section 5.2, the right-hand side $f$ becomes

$$
-\operatorname{div}\left(\rho\left(\left|\nabla u^{\text {int }}\right|\right) \nabla u^{\text {int }}\right)=f,
$$

where

$$
f(r, \varphi):=-\frac{48}{343} r^{-13 / 7} \frac{\sin \left(\frac{4}{7} \varphi\right)}{\left(1+\frac{4}{7} r^{-3 / 7}\right)^{2}} .
$$

Figures 7 and 8 provide the experimental convergence results. As before, the observations are the same as for the linear experiment in Section 5.1. Figure 9 shows some adaptively generated meshes which show a strong mesh-refinement towards the reentrant corner.

\section{Appendix A. Uniform Ellipticity of Discrete Dirichlet-to-Neumann maP}

In [13], Section 3, it is proven that uniform ellipticity of the discrete Dirichlet-to-Neumann $\mathfrak{S}_{\ell}$ implies unique solvability of the discrete problem (2.14) as well as the quasi-optimality (2.17) of discrete solutions. Moreover, [13] prove that $\mathfrak{S}_{\ell}$ is uniformly elliptic if the mesh-width $h_{0}$ of the initial meshes $\mathcal{T}_{0}$ and $\mathcal{E}_{0}$ is sufficiently small. In this appendix, we improve this result and prove that uniform ellipticity of $\mathfrak{S}_{\ell}$ holds without further restrictions on the mesh, i.e. [13], Assumption 1, is not necessary.

To this end, we first recall the necessary definitions: let $X_{\ell}$ be a closed subspace of $H^{1}(\Omega)$ and $Y_{\ell}$ be a closed subspace of $H^{-1 / 2}(\Gamma)$. We assume that the constant functions belong to $Y_{\ell}$, i.e., $1 \in Y_{\ell}$. With the integral operators $\mathfrak{V}, \mathfrak{K}$, and $\mathfrak{W}$, the trace operator $\gamma: H^{1}(\Omega) \rightarrow H^{1 / 2}(\Gamma)$, and the canonical inclusions

$$
i_{\ell}: X_{\ell} \hookrightarrow H^{1}(\Omega) \quad \text { and } \quad j_{\ell}: Y_{\ell} \hookrightarrow H^{-1 / 2}(\Gamma),
$$



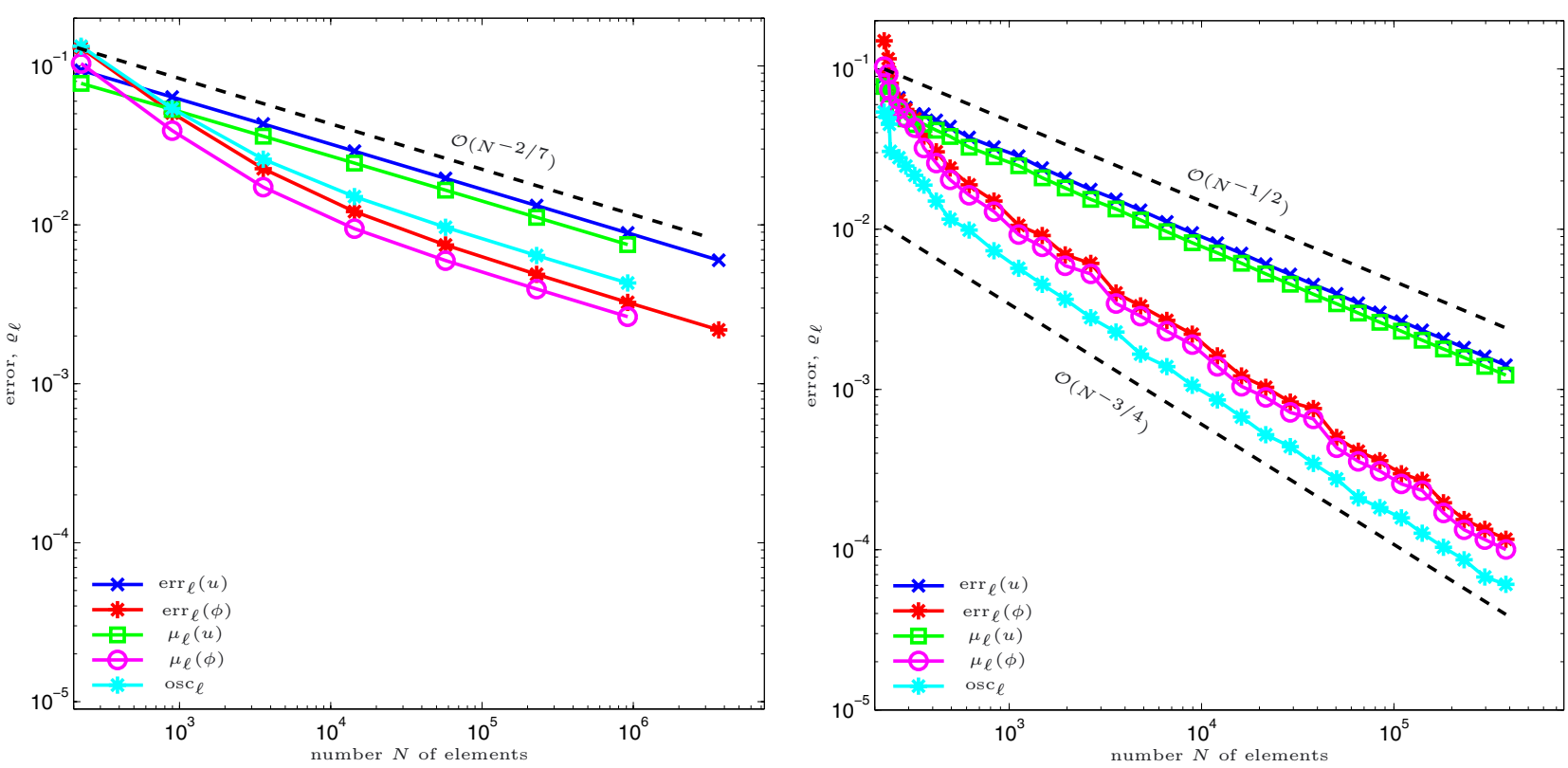

FiguRE 7. Estimators $\operatorname{err}_{\ell}(u), \operatorname{err}_{\ell}(\phi), \mu_{\ell}(u)$ and $\mu_{\ell}(\phi)$ from (5.1)-(5.2) as well as data oscillations osc $_{\ell}$ in nonlinear Experiment 5.3 on the Z-shaped domain, plotted over the number $N=\# \mathcal{T}_{\ell}$ of triangles for uniform (left) and adaptive mesh-refinement (right).
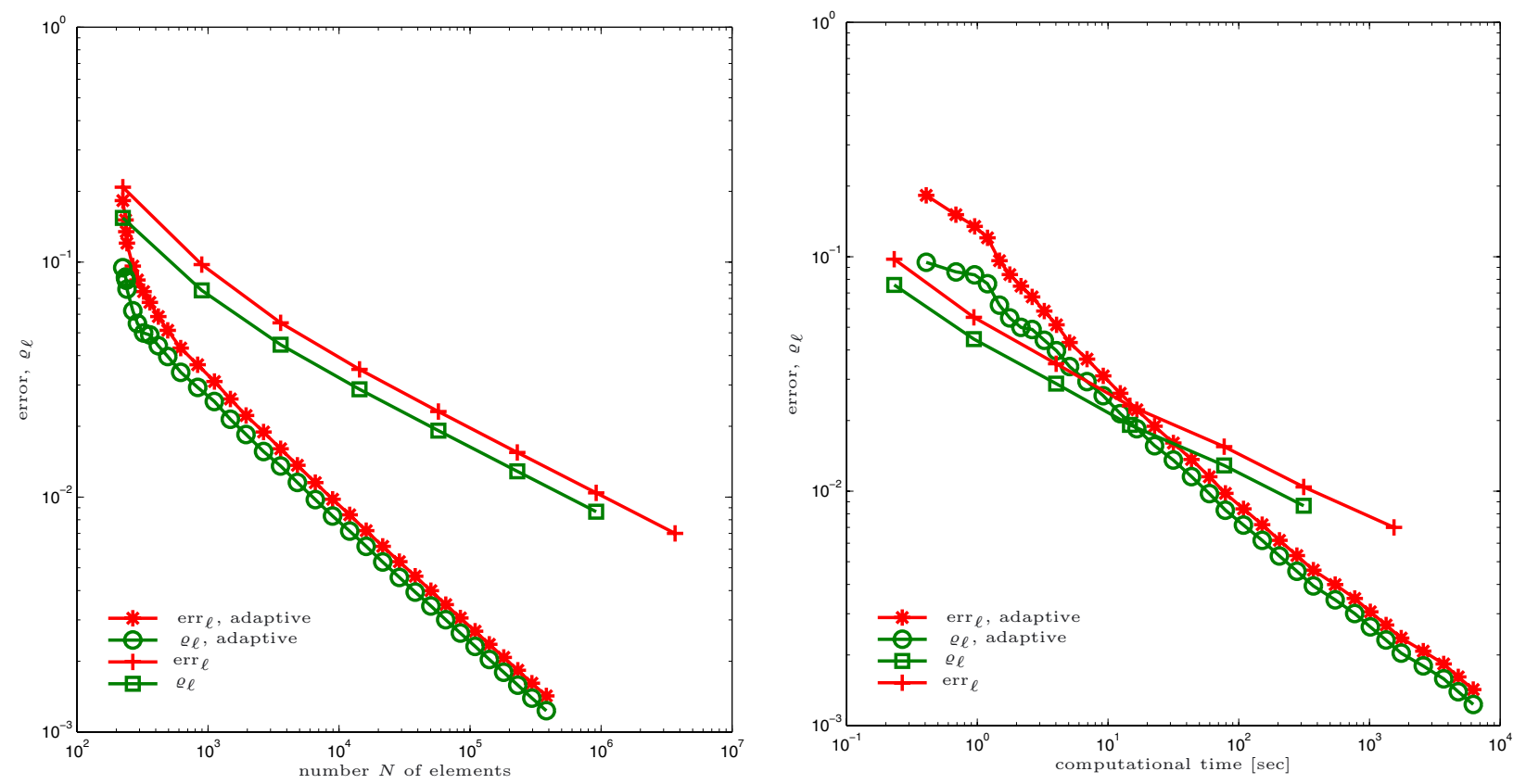

FIgURE 8. Comparison of uniform and adaptive mesh-refining in nonlinear experiment 5.3 on the Z-shaped domain, where the error bound $\operatorname{err}_{\ell}$ and the estimator $\varrho_{\ell}$ from (5.5) are plotted over the number $N=\# \mathcal{T}_{\ell}$ of triangles (left) and over the computational time (right). 

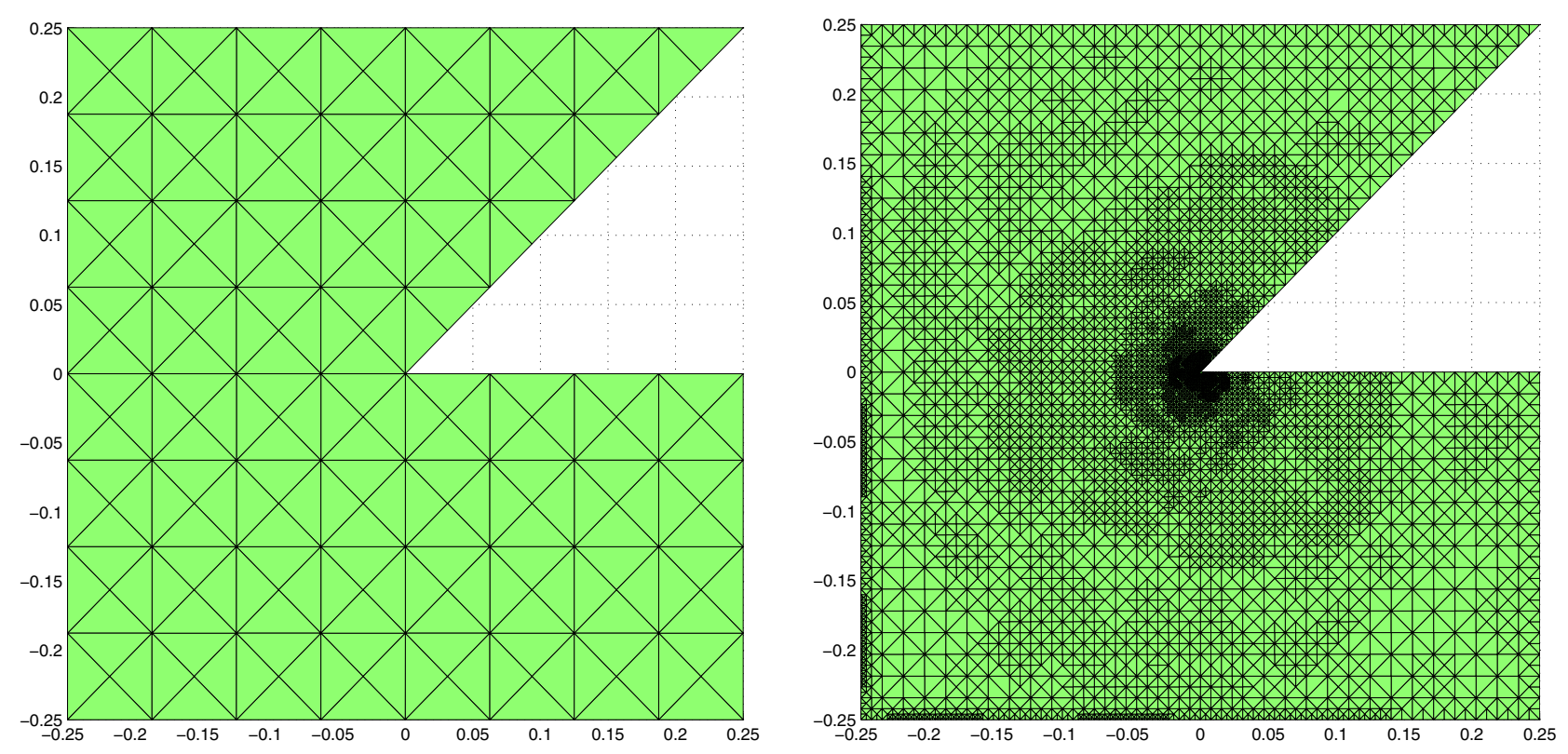

FiguRE 9. Adaptively generated meshes $\mathcal{T}_{\ell}$ in nonlinear experiment 5.3 on the Z-shaped domain.

we formally define the following discrete boundary integral operators

$$
\begin{aligned}
\mathfrak{V}_{\ell} & :=j_{\ell}^{*} \mathfrak{V} j_{\ell}: Y_{\ell} \rightarrow Y_{\ell}^{*}, \\
\mathfrak{K}_{\ell} & :=j_{\ell}^{*} \mathfrak{K} \gamma i_{\ell}: X_{\ell} \rightarrow Y_{\ell}^{*}, \\
\mathfrak{W}_{\ell} & :=i_{\ell}^{*} \gamma^{*} \mathfrak{W} \gamma i_{\ell}: X_{\ell} \rightarrow X_{\ell}^{*} .
\end{aligned}
$$

Moreover, we define some discrete identity

$$
I_{\ell}=j_{\ell}^{*} \gamma i_{\ell}: X_{\ell} \rightarrow Y_{\ell}^{*}
$$

In analogy to (2.9), the discrete Dirichlet-to-Neumann map now reads

$$
\mathfrak{S}_{\ell}:=\mathfrak{W}_{\ell}+\left(\frac{1}{2} I_{\ell}^{*}-\mathfrak{K}_{\ell}^{*}\right) \mathfrak{V}_{\ell}^{-1}\left(\frac{1}{2} I_{\ell}-\mathfrak{K}_{\ell}\right) .
$$

The first elementary lemma states that $\mathfrak{V}_{\ell}$ is continuous and elliptic and that neither of these bounds depend on $\left(X_{\ell}, Y_{\ell}\right)$. In particular, $\mathfrak{V}_{\ell}$ is invertible, and $\mathfrak{S}_{\ell}$ is well-defined.

Lemma A.1. Let $C_{16}, C_{17}>0$ denote the ellipticity constant and the operator norm of the simple-layer potential $\mathfrak{V}$. Then, there holds, for all $\Phi_{\ell} \in Y_{\ell}$,

$$
\left\|\mathfrak{V}_{\ell} \Phi_{\ell}\right\|_{Y_{\ell}^{*}} \leq C_{17}\left\|\Phi_{\ell}\right\|_{H^{-1 / 2}(\Gamma)} \quad \text { as well as } \quad\left\langle\mathfrak{V}_{\ell} \Phi_{\ell}, \Phi_{\ell}\right\rangle \geq C_{16}\left\|\Phi_{\ell}\right\|_{H^{-1 / 2}(\Gamma)}^{2} .
$$

In particular, the lemma of Lax-Milgram applies and proves that $\mathfrak{V}_{\ell}$ is an isomorphism. Moreover, with $C_{18}:=$ $C_{16} / C_{17}^{2}$ holds

$$
\left\langle Z_{\ell}, \mathfrak{V}_{\ell}^{-1} Z_{\ell}\right\rangle \geq C_{18}\left\|Z_{\ell}\right\|_{Y_{\ell}^{*}}^{2} \text { for all } Z_{\ell} \in Y_{\ell}^{*}
$$


Proof. The elementary proof follows from $j_{\ell} \Phi_{\ell}=\Phi_{\ell}$ and $\left\|j_{\ell}^{*}\right\|=\left\|j_{\ell}\right\|=1$ for the operator norms, and (A.2) is an immediate consequence of (A.1).

The following proposition is the main result of this appendix and obtained by boot-strapping of [13], Lemma 3.6.

Proposition A.2. There is a constant $C_{6}>0$ which depends only on $\Omega$ such that for all closed subspaces $X_{\ell}$ of $H^{1}(\Omega)$ and $Y_{\ell}$ of $H^{-1 / 2}(\Gamma)$ with $1 \in Y_{\ell}$, there holds

$$
\left\langle\mathfrak{S}_{\ell} U_{\ell}, U_{\ell}\right\rangle \geq C_{19}\left\|\gamma U_{\ell}\right\|_{H^{1 / 2}(\Gamma)}^{2} \quad \text { for all } U_{\ell} \in X_{\ell} .
$$

Proof. We aim at proving the following claim:

$$
\exists C_{19}>0 \forall\left(X_{\ell}, Y_{\ell}\right)_{\ell \in \mathbb{N}} \text { with } 1 \in Y_{\ell} \forall \ell \in \mathbb{N} \forall U_{\ell} \in X_{\ell} \quad\left\langle\mathfrak{S}_{\ell} U_{\ell}, U_{\ell}\right\rangle \geq C_{19}\left\|\gamma U_{\ell}\right\|_{H^{1 / 2}(\Gamma)}^{2} .
$$

By choosing the constant sequence of spaces $\left(X_{\ell}, Y_{\ell}\right)$, we see that (A.4) implies (A.3). To prove (A.4), we argue by contradiction and assume that (A.4) is wrong, i.e.,

$$
\forall c>0 \exists\left(X_{\ell}, Y_{\ell}\right)_{\ell \in \mathbb{N}} \text { with } 1 \in Y_{\ell} \exists \ell \in \mathbb{N} \exists U_{\ell} \in X_{\ell} \quad\left\langle\mathfrak{S}_{\ell} U_{\ell}, U_{\ell}\right\rangle<c\left\|\gamma U_{\ell}\right\|_{H^{1 / 2}(\Gamma)}^{2} .
$$

For $\ell \in \mathbb{N}$ and $c=1 / \ell$, we may therefore choose some subspaces $X_{\ell}$ of $H^{1}(\Omega)$ and $Y_{\ell}$ of $H^{-1 / 2}(\Gamma)$ with $1 \in Y_{\ell}$ as well as some $U_{\ell} \in X_{\ell}$ such that

$$
\left\langle\mathfrak{S}_{\ell} U_{\ell}, U_{\ell}\right\rangle<\frac{1}{\ell}\left\|\gamma U_{\ell}\right\|_{H^{1 / 2}(\Gamma)}^{2}
$$

In particular, this yields $\gamma U_{\ell} \neq 0$ and hence

$$
\left\langle\mathfrak{S}_{\ell} V_{\ell}, V_{\ell}\right\rangle<\frac{1}{\ell} \quad \text { with } \quad V_{\ell}:=\frac{U_{\ell}}{\left\|\gamma U_{\ell}\right\|_{H^{1 / 2}(\Gamma)}} \in X_{\ell} \quad \text { for all } \ell \in \mathbb{N}
$$

Since $\left(\gamma V_{\ell}\right)_{n \in \mathbb{N}}$ is bounded in $H^{1 / 2}(\Gamma)$, we may assume - without loss of generality - that there holds weak convergence

$$
\gamma V_{\ell} \rightarrow v \in H^{1 / 2}(\Gamma) \quad \text { as } \ell \rightarrow \infty
$$

In the following, we will now show that $\gamma V_{\ell}$ converges to $v$ even strongly in $H^{1 / 2}(\Gamma)$ and that $v \neq 0$ is constant.

First, the definition of $\mathfrak{S}_{\ell}$ and the $\ell$-independent ellipticity of $\mathfrak{V}_{\ell}^{-1}$ give

$$
\begin{aligned}
\left\langle\mathfrak{S}_{\ell} V_{\ell}, V_{\ell}\right\rangle & =\left\langle\mathfrak{W} \gamma V_{\ell}, \gamma V_{\ell}\right\rangle+\left\langle\mathfrak{V}_{\ell}^{-1}\left(\frac{1}{2} I_{\ell}-\mathfrak{K}_{\ell}\right) V_{\ell},\left(\frac{1}{2} I_{\ell}-\mathfrak{K}_{\ell}\right) V_{\ell}\right\rangle \\
& \geq\left\langle\mathfrak{W} \gamma V_{\ell}, \gamma V_{\ell}\right\rangle+C_{18}\left\|\left(\frac{1}{2} I_{\ell}-\mathfrak{K}_{\ell}\right) V_{\ell}\right\|_{Y_{\ell}^{*}}^{2} .
\end{aligned}
$$

Note that the hypersingular integral operator is positive semi-definite. Therefore, the right-hand side is nonnegative, and (A.5) proves that both terms on the right-hand side tend to zero. Note that the functional

$$
H^{1 / 2}(\Gamma) \rightarrow \mathbb{R}, u \mapsto\langle\mathfrak{W} u, u\rangle
$$

is continuous and convex, whence weakly lower semicontinuous. With (A.6), this implies

$$
\langle\mathfrak{W} v, v\rangle \leq \liminf _{\ell \rightarrow \infty}\left\langle\mathfrak{W} \gamma V_{\ell}, \gamma V_{\ell}\right\rangle=0 .
$$


Consequently, the weak limit $v \in H^{1 / 2}(\Gamma)$ is constant. This and (A.6) imply

$$
\frac{1}{|\Gamma|} \int_{\Gamma} \gamma V_{\ell} \mathrm{d} s \stackrel{\ell \rightarrow \infty}{\longrightarrow} \frac{1}{|\Gamma|} \int_{\Gamma} v \mathrm{~d} s=v
$$

Note that an equivalent norm on $H^{1 / 2}(\Gamma)$ is given by

$$
\|u\|_{H^{1 / 2}(\Gamma)} \simeq\|u\|:=\left(\langle\mathfrak{W} u, u\rangle+\left|\int_{\Gamma} u \mathrm{~d} s\right|^{2}\right)^{1 / 2} \quad \text { for } u \in H^{1 / 2}(\Gamma) .
$$

We now define

$$
w_{\ell}:=\gamma V_{\ell}-\frac{1}{|\Gamma|} \int_{\Gamma} \gamma V_{\ell} \mathrm{d} s
$$

By definition, there holds

$$
\left\|w_{\ell}\right\|^{2}=\left\langle\mathfrak{W} w_{\ell}, w_{\ell}\right\rangle=\left\langle\mathfrak{W} \gamma V_{\ell}, \gamma V_{\ell}\right\rangle \stackrel{\ell \rightarrow \infty}{\longrightarrow} 0,
$$

i.e. there holds strong convergence $w_{\ell} \rightarrow 0 \in H^{1 / 2}(\Gamma)$. Consequently, we now obtain

$$
\left\|\gamma V_{\ell}-v\right\|_{H^{1 / 2}(\Gamma)} \leq\left\|w_{\ell}\right\|_{H^{1 / 2}(\Gamma)}+\left|\frac{1}{|\Gamma|} \int_{\Gamma} \gamma V_{\ell} \mathrm{d} s-v\right||\Gamma|^{1 / 2} \stackrel{\ell \rightarrow \infty}{\longrightarrow} 0,
$$

i.e. we have proven that $\gamma V_{\ell}$ converges strongly to the constant $v$ in $H^{1 / 2}(\Gamma)$. In particular, the norm convergence yields

$$
\|v\|_{H^{1 / 2}(\Gamma)}=\lim _{\ell \rightarrow \infty}\left\|\gamma V_{\ell}\right\|_{H^{1 / 2}(\Gamma)}=1,
$$

i.e. the limit of $\gamma V_{\ell}$ is a constant $v \neq 0$.

Since $v$ is constant, there holds $\mathfrak{K} v=-v / 2$, whence

$$
0 \neq v\langle 1,1\rangle=\lim _{\ell \rightarrow \infty}\left\langle j_{\ell} 1,\left(\frac{1}{2}-\mathfrak{K}\right) \gamma V_{\ell}\right\rangle=\lim _{\ell \rightarrow \infty}\left\langle 1,\left(\frac{1}{2} I_{\ell}-\mathfrak{K}_{\ell}\right) V_{\ell}\right\rangle
$$

where we have used that $1 \in Y_{\ell}$. However, we have already observed above that

$$
\left|\left\langle 1,\left(\frac{1}{2} I_{\ell}-\mathfrak{K}_{\ell}\right) V_{\ell}\right\rangle\right| \leq\|1\|_{H^{-1 / 2}(\Gamma)}\left\|\left(\frac{1}{2} I_{\ell}-\mathfrak{K}_{\ell}\right) V_{\ell}\right\|_{Y_{\ell}^{*}} \stackrel{\ell \rightarrow \infty}{\longrightarrow} 0 .
$$

This contradiction concludes the proof.

Acknowledgements. The research of the authors is supported through the FWF project Adaptive Boundary Element Method, funded by the Austrian Science Fund (FWF) under grant P21732.

\section{REFERENCES}

[1] M. Ainsworth and J.T. Oden, A posteriori error estimation in finite element analysis. Wiley-Interscience, John Wiley \& Sons, New-York (2000).

[2] M. Aurada, P. Goldenits and D. Praetorius, Convergence of data perturbed adaptive boundary element methods. ASC Report 40/2009, Institute for Analysis and Scientific Computing, Vienna University of Technology, Wien (2009). 
[3] M. Aurada, M. Ebner, M. Feischl, S. Ferraz-Leite, P. Goldenits, M. Karkulik, M. Mayr and D. Praetorius, HILBERT - A Matlab implementation of adaptive 2D-BEM. ASC Report 24/2011, Institute for Analysis and Scientific Computing, Vienna University of Technology, Wien (2011). Software download at http://www.asc.tuwien.ac.at/abem/hilbert/.

[4] M. Aurada, S. Ferraz-Leite and D. Praetorius, Estimator reduction and convergence of adaptive BEM. Appl. Numer. Math., in print (2011).

[5] I. Babuśka and M. Vogelius, Feedback and adaptive finite element solution of one-dimensional boundary value problems. Numer. Math. 44 (1984) 75-102.

[6] R. Bank, Hierarchical bases and the finite element method. Acta Numer. 5 (1996) 1-45.

[7] F. Bornemann, B. Erdmann and R. Kornhuber, A-posteriori error-estimates for elliptic problems in 2 and 3 space dimensions. SIAM J. Numer. Anal. 33 (1996) 1188-1204.

[8] C. Carstensen, An a posteriori error estimate for a first-kind integral equation. Math. Comp. 66 (1997) $139-155$.

[9] C. Carstensen and D. Praetorius, Averaging techniques for the effective numerical solution of Symm's integral equation of the first kind. SIAM J. Sci. Comput. 27 (2006) 1226-1260.

[10] C. Carstensen and D. Praetorius, Averaging techniques for the a posteriori BEM error control for a hypersingular integral Equation in two dimensions. SIAM J. Sci. Comput. 29 (2007) 782-810.

[11] C. Carstensen and D. Praetorius, Averaging techniques for a posteriori error control in finite element and boundary element analysis, in Boundary Element Analysis: Mathematical Aspects and Applications, edited by M. Schanz and O. Steinbach. Lect. Notes Appl. Comput. Mech. 29 (2007) 29-59.

[12] C. Carstensen and D. Praetorius, Convergence of adaptive boundary element methods. ASC Report 15/2009, Institute for Analysis and Scientific Computing, Vienna University of Technology, Wien (2009).

[13] C. Carstensen and E. Stephan, Adaptive coupling of boundary elements and finite elements. ESAIM: M2AN 29 (1995) $779-817$.

[14] M. Costabel, A symmetric method for the coupling of finite elements and boundary elements, in The Mathematics of Finite Elements and Applications IV, MAFELAP 1987, edited by J. Whiteman, Academic Press, London (1988) $281-288$.

[15] P. Deuflhard, P. Leinen and H. Yserentant, Concepts of an adaptive hierarchical finite element code. Impact Comput. Sci. Eng. 1 (1989) 3-35.

[16] W. Dörfler, A convergent adaptive algorithm for Poisson's equation. SIAM J. Numer. Anal. 33 (1996) 1106-1124.

[17] W. Dörfler and R. Nochetto, Small data oscillation implies the saturation assumption. Numer. Math. 91 (2002) 1-12.

[18] C. Erath, S. Ferraz-Leite, S. Funken and D. Praetorius, Energy norm based a posteriori error estimation for boundary element methods in two dimensions. Appl. Numer. Math. 59 (2009) 2713-2734.

[19] C. Erath, S. Funken, P. Goldenits and D. Praetorius, Simple error estimators for the Galerkin BEM for some hypersingular integral equation in 2D. ASC Report 20/2009, Institute for Analysis and Scientific Computing, Vienna University of Technology, Wien (2009).

[20] S. Ferraz-Leite and D. Praetorius, Simple a posteriori error estimators for the h-version of the boundary element method. Computing 83 (2008) 135-162.

[21] S. Ferraz-Leite, C. Ortner and D. Praetorius, Convergence of simple adaptive Galerkin schemes based on $h-h / 2$ error estimators. Numer. Math. 116 (2010) 291-316.

[22] I. Graham, W. Hackbusch and S. Sauter, Finite elements on degenerate meshes: Inverse-type inequalities and applications. IMA J. Numer. Anal. 25 (2005) 379-407.

[23] E. Hairer, S. Nørsett and G. Wanner, Solving ordinary differential equations I, Nonstiff problems. Springer, New York (1987).

[24] M. Maischak, P. Mund and E. Stephan, Adaptive multilevel BEM for acoustic scattering. Comput. Methods Appl. Mech. Eng. 150 (2001) 351-367.

[25] W. McLean, Strongly elliptic systems and boundary integral equations. Cambridge University Press, Cambridge (2000).

[26] P. Morin, K. Siebert and A. Veeser, A Basic convergence result for conforming adaptive finite elements. Math. Models Methods Appl. Sci. 18 (2008) 707-737.

[27] P. Mund and E. Stephan, An additive two-level method for the coupling of nonlinear FEM-BEM equations. SIAM J. Numer. Anal. 36 (1999) 1001-1021.

[28] P. Mund, E. Stephan and J. Weiße, Two-level methods for the single layer potential in $\mathbb{R}^{3}$. Computing 60 (1998) 243-266.

[29] S. Rjasanov and O. Steinbach, The fast solution of boundary integral equations. Springer, New York (2007).

[30] S. Sauter and C. Schwab, Randelementmethoden: Analyse, Numerik und Implementierung schneller Algorithmen. Teubner Verlag, Wiesbaden (2004).

[31] O. Steinbach, Numerical approximation methods for elliptic boundary value problems: Finite and boundary elements. Springer, New York (2008).

[32] R. Verfürth, A review of a posteriori error estimation and adaptive mesh-refinement techniques. Teubner, Stuttgart (1996).

[33] E. Zeidler, Nonlinear functional analysis and its applications. part II/B, Springer, New York (1990). 